\title{
Linx
}

Revue des linguistes de l'université Paris X Nanterre

$82 \mid 2021$

Entre vieillissement et innovation : le changement linguistique

\section{Un déclin différé : trajectoires de deux traits conservateurs du français québécois au cours du $20^{\mathrm{e}}$ siècle}

Delayed decline: trajectories of two conservative features of Quebec French

throughout the 20th century

\section{Hugo Saint-Amant Lamy}

\section{CpenEdition}

Journals

Édition électronique

URL : https://journals.openedition.org/linx/8148

DOI : 10.4000/linx.8148

ISSN : 2118-9692

\section{Éditeur}

Presses universitaires de Paris Nanterre

Référence électronique

Hugo Saint-Amant Lamy, « Un déclin différé : trajectoires de deux traits conservateurs du français québécois au cours du 20e siècle », Linx [En ligne], 82 | 2021, mis en ligne le 15 juillet 2021, consulté le 21 juillet 2021. URL : http://journals.openedition.org/linx/8148; DOI : https://doi.org/10.4000/linx. 8148

Ce document a été généré automatiquement le 21 juillet 2021.

Département de Sciences du langage, Université Paris Ouest 


\title{
Un déclin différé : trajectoires de deux traits conservateurs du français québécois au cours du $20^{\mathrm{e}}$ siècle ${ }^{1}$
}

\author{
Delayed decline: trajectories of two conservative features of Quebec French \\ throughout the 20th century
}

Hugo Saint-Amant Lamy

\section{Introduction}

1 En 1763, le Traité de Paris marque la fin de la guerre de Sept Ans et officialise la cession des colonies françaises nord-américaines à la Grande-Bretagne. La rupture qui s'opère alors entre la France et le Québec aura des conséquences linguistiques profondes, dont le maintien en Amérique de certains traits phonologiques qui, déjà au $18^{\mathrm{e}}$ siècle, tombent en désuétude dans la région parisienne. Certains de ces traits persisteront au Québec jusqu'aux $20^{\mathrm{e}}$ et $21^{\mathrm{e}}$ siècles, où leur déclin peut de nos jours être mesuré avec des méthodes phonétiques auparavant inaccessibles. La présente étude propose une analyse acoustique de deux de ces traits conservateurs du français québécois : la réalisation antérieure de la diphtongue -oi devant $/ \mathrm{B} /$ final et le maintien de l'opposition entre /e/ et /3/ devant / $/$ / final.

2 La variante de la diphtongue -oi traditionnellement en usage au Québec devant un /в/ final est [w3] (еx. poire [ртзь]). Celle-ci a toutefois aujourd'hui largement été remplacée par la variante basse [wo] (ех. ршрь) ${ }^{2}$. Le changement est analogue à celui survenu en France quelques siècles plus tôt, où la variante $[\mathrm{w} \varepsilon]$ a été supplantée par [wa]. Le français québécois a également longtemps maintenu les /e/ paroxytoniques du moyen français dans les mots ayant la graphie -(i)ère (Morin, 2009). Ce /e/ s'opposait à un $/ 3 /$ pré-rhotique, d'où des paires minimales comme père $[\mathrm{per}]-$ paire [рзв]. Cette 
opposition s'est aujourd'hui largement neutralisée en faveur de la variante mi-ouverte, suivant la tendance tracée en France précédemment.

Les deux phénomènes sont analysés à partir d'entretiens guidés effectués auprès de locuteurs nés entre 1872 et 1997, et ce dans le cadre de deux projets géolinguistiques québécois: l'Atlas linguistique de l'est du Canada (Dulong et Bergeron, 1980) et Phonologie du français contemporain (Durand, Laks et Lyche, 2014 ; Côté, 2014).

4 L'objectif général de l'étude est de clarifier les liens qui existent entre le déclin des formes conservatrices au Québec, et celui observé précédemment en France, et de déterminer à quel point le premier peut être considéré comme l'extension du second. Les schémas temporels et spatiaux qui caractérisent l'abandon de la diphtongue [w3] et $\mathrm{du}[\mathrm{e}]$ pré-rhotiques sont d'abord analysés. Une attention particulière est également portée à la nature, continue ou catégorique, de la transition entre formes anciennes et nouvelles. Enfin, un certain nombre de facteurs de variation rapportés par des auteurs français des $17^{\mathrm{e}}$ et $18^{\mathrm{e}}$ siècles sont é valués dans le contexte québécois.

Une brève histoire du discours normatif entourant la réalisation de la diphtongue -oi et l'opposition $/ \mathrm{e} /-/ \varepsilon /$ devant $/ \mathrm{B} /$ est présentée à la section 2 . La méthodologie employée est détaillée à la section 3 . Les sections 4 et 5 sont respectivement consacrées aux résultats obtenus pour les deux phénomènes. L'étude se termine avec une analyse des résultats à la section 6, ainsi qu'une conclusion générale à la section 7.

\section{Le déclin de [wع] et [e] devant / $\mathrm{b} /$}

6 Les normes de prononciation relatives à la diphtongue -oi et à l'opposition /e/-/3/ devant $/ \mathrm{B} /$ connaissent toutes deux un renversement en France au cours des $17^{\mathrm{e}}$ et $18^{\mathrm{e}}$ siècles. Une compilation par Thurot (1881) de témoignages portant sur la prononciation $\mathrm{du}$ français permet de déterminer approximativement la chronologie de ces renversements. L'absence d'une résolution normative pour les deux phénomènes avant la cession de la Nouvelle-France (1763) permet aux variantes conservatrices de se maintenir plus longtemps au Québec. Ce n'est qu'à partir du milieu du $19^{\mathrm{e}}$ siècle que celles-ci y sont explicitement condamnées.

\subsection{Changement de norme en France aux $17^{\mathrm{e}}$ et $18^{\mathrm{e}}$ siècles}

7 La variante [wa] de la diphtongue apparaît chez le «bas peuple de Paris » dès le $14^{\mathrm{e}}$ siècle (Pasques, 1975), mais il faut attendre le $17^{\mathrm{e}}$ siècle avant qu'elle n'entre en concurrence avec $[w \varepsilon]$ dans le discours normatif. Les points de vue des auteurs cités par Thurot sur le sujet sont classés de façon schématique à la Figure 1. Des 41 auteurs cités, 22 ne reconnaissent que la prononciation $[\mathrm{w} \varepsilon]^{3}, 11$ ne reconnaissent que le [wa] ${ }^{4}$ et 4 relèvent explicitement des cas de variation. De Longue (1725) et De la Lande (1730) rapportent que la prononciation [wa] est utilisée dans les verbes à l'infinitif, et que [wE] l'est ailleurs. Galmace (1767) préconise l'usage de [wE], mais reconnaît que «des personnes instruites et d'un rang distingué soutiennent qu'on doit prononcer roua, loua, savouar » (Thurot, $1881: 361$ ). Enfin, Durand (1767) soutient que l'on devrait entendre « un son intermédiaire entre $a$ et $\grave{e} »^{5}$ (Thurot, $1881: 361$ ). 
Si seul [wع] est recommandé avant le $17^{\mathrm{e}}$ siècle, il ne l'est plus du tout à partir de la décennie 1770. La période de variation normative entre [wع] et [wa] correspond donc exactement à celle du Régime français au Canada (1608 - 1763).

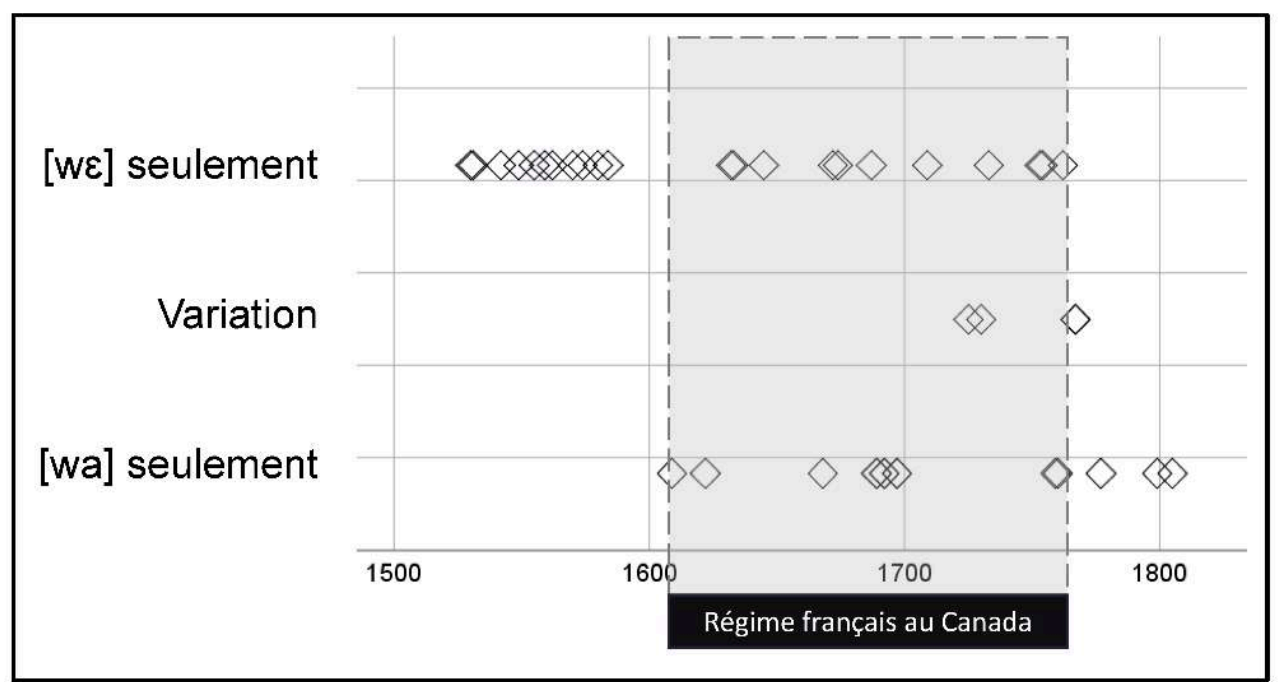

Figure 1. Diphtongue -oi : évolution des positions normatives

L'opposition phonémique /e/-/ $/$ / en syllabe accentuée remonte au moins à l'ancien français primitif (Morin, 2009). Sa neutralisation commence vraisemblablement bien avant que l'on commence à la prescrire. Les recommandations des différents auteurs cités par Thurot sont résumées à la Figure 2. Des 35 auteurs cités, 12 préconisent l'usage de $[e]$ pour les mots ayant la graphie -(i)ère ${ }^{6}, 7$ suggèrent plutôt le $[\varepsilon]^{7}$ et 16 relèvent des cas de variation. Les hypothèses quant au conditionnement de cette variation sont nombreuses. Plusieurs auteurs avancent que la voyelle dans les séquences -(i)ère se situe à mi-chemin entre $[\mathrm{e}]$ et $[\varepsilon]$ (Mourgues, 1685 ; Dangeau, 1694; Grimarest, 1712 ; Restaut, 1730 ; D'Olivet, 1767 ; Demandre, 1769). D'autres suggèrent que l'on trouve la voyelle mi-fermée $[\mathrm{e}]$ dans les séquences -ière et la mi-ouverte $[\varepsilon]$ dans les séquences ère (Saint-Liens, 1580 ; Dobert, 1650 ; D'Allais, 1681; Hindret, 1687). L'existence de classes lexicales distinctes est évoquée. Par exemple, Martin (1632) préconise le [e] dans lumière, mais le $[\varepsilon]$ dans jartière (sic). Selon Oudin (1633) et Chifflet (1659), on trouverait la mi-fermée dans les mots père, mère, frère et leurs dérivés (ex. compère), mais la miouverte partout ailleurs. Hindret (1687) rapporte [e] dans les adjectifs (ex. chère, légère), mais $[\varepsilon]$ dans les autres mots en -ère. Certains cas de variation géographique sont également signalés. Buffier note que les Normands prononcent « pére, mére » (Thurot, 1881 : 73). Les Picards prononceraient également le mot père avec une voyelle mifermée selon Montmignon (1785). Enfin, le cas le plus intéressant de variation est peutêtre celui de la $3^{\mathrm{e}}$ édition du Dictionnaire de l'Académie (1740) où, " après le mot misére, on ne rencontre plus de mots en -ere qu'écrits avec un $e$ ouvert, et qu'avant ce mot l'e ouvert est exceptionnel. On avait sans doute changé d'avis dans le cours de l'impression» (Thurot, 1881: 74). Ce renversement spectaculaire illustre bien les changements normatifs importants qui ont cours dans les années précédant la Révolution.

Avant le $17^{\mathrm{e}}$ siècle, la voyelle [e] est nettement favorisée dans les séquences-(i)ère. Les deux siècles qui suivent, qui correspondent grosso modo au Régime français au Canada, 
sont toutefois marqués par une très importante variation normative, qui se résout éventuellement vers la forme $[\varepsilon]$.

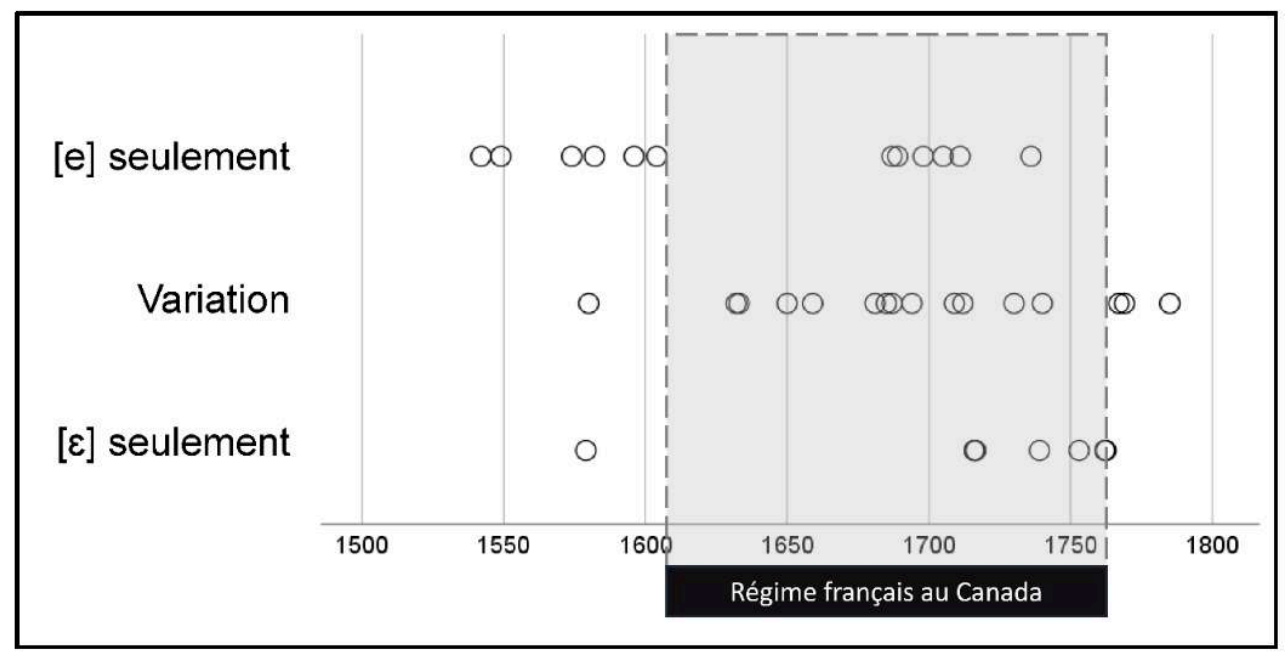

Figure 2. Classe vocalique /e/ : évolution des positions normatives

\subsection{Changement de norme au Québec aux $19^{\mathrm{e}}$ et $20^{\mathrm{e}}$ siècles}

Au Québec, la question de la légitimité des différentes variantes de la diphtongue -oi fait l'objet d'une polémique au milieu du $19^{\mathrm{e}}$ siècle (Dionne, 1912 ; Bouchard, 2011). Si les participants reconnaissent que la variante [wE] est largement utilisée par leurs concitoyens, certains préconisent un alignement sur la nouvelle norme hexagonale. C'est le cas de Maguire (1841) et Bibaud (1842), qui se basent sur des ouvrages français et la fréquentation de prêtres arrivés au Canada après la Révolution. D’autres, comme Demers (1842), qui se basent sur des ouvrages français n'ayant pas adopté la nouvelle forme [wa], défendent la variante traditionnelle. Le désaccord persiste jusqu'au début $\mathrm{du} 20^{\mathrm{e}}$ siècle, où de rares auteurs continuent à défendre la variante [wE] (Paris, 1901 ; Tardivel, 1901). La réalisation [wa] s'impose toutefois dans les manuels de diction ultérieurs (Rivard, 1901; Dumais, 1905; Hudon, 1931; Landreau, 1931).

La neutralisation de /е/-/ع/ devant / $/$ / fait l'objet de condamnations moins univoques que les différentes réalisations de la diphtongue -oi. Très peu d'auteurs prennent le temps de proscrire explicitement le [e] pré-rhotique. Maguire (1841:173-175) n'aborde pas le sujet ouvertement, mais emploie la graphie «ère » dans certaines transcriptions phonétiques (aiguillère : « é-ghi-ère »; équilatère : « é-ku-i-latère »). Manseau (1881:110) est un peu plus direct lorsqu'il affirme que le mot prononcé « ayére » (œillère) doit être prononcé « eu-yère ». Rinfret (1896: 281) condamne explicitement l'usage de [e] devant [3] (ex. neige, piège, collège), mais pas devant /ь/. Malgré la subtilité de la proscription $\mathrm{du}[\mathrm{e}]$ devant $/ \mathrm{B} /$, on observe son intégration chez une partie de la population. À la fin $\mathrm{du} 19^{\mathrm{e}}$ siècle, Fréchette rapporte qu'il a « entendu une dame se moquer de quelqu'un qui disait mon pére, mon frére » (Fréchette, $1894: 1$ ). Les manuels de diction du début du $20^{\mathrm{e}}$ siècle préconisent catégoriquement la réalisation mi-ouverte (Rivard, 1901; Dumais, 1905; Hudon, 1931; Landreau, 1931).

13 Que ce soit dans le cas de la diphtongue -oi ou celui de la neutralisation de /e/-/E/, on observe un changement du discours normatif à partir de la seconde moitié du $19^{e}$ siècle. 
On connaît toutefois encore mal l'évolution que l'usage a connu en réaction à ces différentes prescriptions.

\section{Méthodologie}

\subsection{Source des données}

L'évolution du système sonore du français québécois au cours du dernier siècle peut être étudiée par la mise en relation de deux ensembles de données géolinguistiques : l'Atlas linguistique de l'est du Canada (ALEC; Dulong et Bergeron, 1980) le projet Phonologie du français contemporain (PFC; Durand, Laks et Lyche, 2014).

L'ALEC s'inscrit dans la tradition dialectologique de l'Atlas linguistique de France. Entre 1969 et 1971, une équipe de quatre enquêteurs visite 169 localités du Québec et des provinces voisines. Si les concepteurs du projet privilégient une approche par questionnaire lexicographique, leur protocole prévoit également un entretien d'une vingtaine de minutes avec un témoin jugé représentatif de chaque communauté. Si une partie des enregistrements sont à ce jour introuvables, plusieurs sont toutefois conservés dans le fonds Dulong des Archives de folklore et d'ethnologie de l'Université Laval.

De son côté, le projet PFC adopte l'approche de la linguistique de corpus afin de documenter la variation linguistique au sein de la francophonie. Son protocole prévoit un échantillon équilibré d'une douzaine de témoins et une série de tâches visant à éliciter une variété de registres : lecture de listes de mots, lectures d'un texte, entretien guidé, conversation libre. À ce jour, 32 enquêtes ont été effectuées au Canada en milieu francophone majoritaire (voir Côté, 2014), pour un total de 447 témoins. Le traitement des enquêtes étant toujours en cours, un sous-échantillon de 16 enquêtes est utilisé ici. Ces enquêtes ont été effectuées entre 2009 et 2016.

17 La mise en relation des enregistrements sonores de l'ALEC et de PFC pose un certain nombre de contraintes qui doivent être contournées par des choix méthodologiques parfois difficiles (voir Saint-Amant Lamy, sous presse). Considérant la sousreprésentation marquée des femmes dans l'ALEC, seules les données des témoins masculins sont utilisées ici. Afin de maintenir une base de comparabilité en ce qui a trait au type de tâche, seuls les entretiens guidés du protocole PFC sont retenus. Enfin, puisque les réseaux d'enquête des deux projets sont différents, les points d'enquête sont réunis en ensembles géographiques plus larges.

18 Le corpus composite obtenu compte 153 témoins masculins (55 de l'ALEC, 98 du souséchantillon PFC) nés entre 1872 et 1997. Les 64 localités représentées sont réunies en six régions géographiques dont la composition est résumée ci-dessous (voir Tableau 1). La carte de ces régions est présentée à la Figure 3. 
Tableau 1. Composition des régions $\left(*\right.$ haute densité; ${ }^{\star *}=$ densité moyenne)

\begin{tabular}{|c|c|c|c|c|}
\hline \multirow{2}{*}{$\begin{array}{l}\text { Régions } \\
\text { Nord-Ouest }\end{array}$} & \multicolumn{2}{|c|}{ Localités ALEC } & \multirow{2}{*}{\begin{tabular}{l}
\multicolumn{1}{c}{ Localités PFC } \\
Gatineau* \\
Hawkesbury**
\end{tabular}} & \multirow{2}{*}{$\begin{array}{c}\begin{array}{c}\mathrm{N}^{\text {bre }} \\
\text { locute }\end{array} \\
19\end{array}$} \\
\hline & $\begin{array}{l}\text { Ferme-Neuve } \\
\text { Masson }\end{array}$ & $\begin{array}{l}\text { St-Jovite } \\
\text { Senneterre }\end{array}$ & & \\
\hline $\begin{array}{l}\text { Région de } \\
\text { Montréal }\end{array}$ & $\begin{array}{l}\text { Lachenaie** } \\
\text { Rigaud } \\
\text { St-Barthélémy }\end{array}$ & $\begin{array}{l}\text { St-Damien } \\
\text { St-Isidore } \\
\text { St-Lin-Laurentides }\end{array}$ & $\begin{array}{l}\text { Montréal* } \\
\text { Ste-Adèle** }^{*}\end{array}$ & 18 \\
\hline Centre & $\begin{array}{l}\text { Baie-du-Febvre } \\
\text { Coaticook** } \\
\text { L'Avenir } \\
\text { St-Étienne-de-Bolton }\end{array}$ & $\begin{array}{l}\text { St-Joseph** } \\
\text { St-Pierre-les-Becquets } \\
\text { Sherbrooke* }\end{array}$ & $\begin{array}{l}\text { St-Tite } \\
\text { Sherbrooke* } \\
\text { Trois-Rivières* } \\
\text { Wickham }\end{array}$ & 31 \\
\hline $\begin{array}{l}\text { Région de } \\
\text { Québec }\end{array}$ & $\begin{array}{l}\text { Arthabaska** } \\
\text { Lac-Mégantic** } \\
\text { St-Augustin } \\
\text { St-Évariste } \\
\text { St-Joseph-de-Beauce }\end{array}$ & $\begin{array}{l}\text { St-Pierre-de-Broughton } \\
\text { St-Ubalde } \\
\text { Ste-Anne-de-Beaupré } \\
\text { Ste-Croix }\end{array}$ & $\begin{array}{l}\text { Québec* } \\
\text { St-Éphrem-de-Beauce }\end{array}$ & 20 \\
\hline Nord-Est & $\begin{array}{l}\text { Baie-Saint-Paul } \\
\text { Grande-Baie } \\
\text { La Malbaie** } \\
\text { Natashquan } \\
\text { Petit-Saguenay }\end{array}$ & $\begin{array}{l}\text { Rivière-au-Tonnerre } \\
\text { Sacré-Cœur } \\
\text { Saint-Félicien } \\
\text { Tête-à-la-Baleine }\end{array}$ & $\begin{array}{l}\text { Baie-Saint-Paul** } \\
\text { Saguenay* } \\
\text { Sept-îles** }\end{array}$ & 30 \\
\hline Sud-Est & $\begin{array}{l}\text { Cap-des-Rosiers } \\
\text { Causapscal } \\
\text { Gaspé** } \\
\text { Les Méchins } \\
\text { Pabos } \\
\text { Petite-Vallée } \\
\text { Rimouski** } \\
\text { Saint-Antonin }\end{array}$ & $\begin{array}{l}\text { Saint-Denis } \\
\text { Sainte-Angèle } \\
\text { Saint-Éleuthère } \\
\text { Saint-Fabien-sur-Mer } \\
\text { Saint-Honoré } \\
\text { Saint-Jean-Port-Joli } \\
\text { Trinité-des-Monts } \\
\text { Val-Brillant }\end{array}$ & $\begin{array}{l}\text { Cap-Chat } \\
\text { La Pocatière** } \\
\text { Rimouski* }\end{array}$ & 35 \\
\hline
\end{tabular}

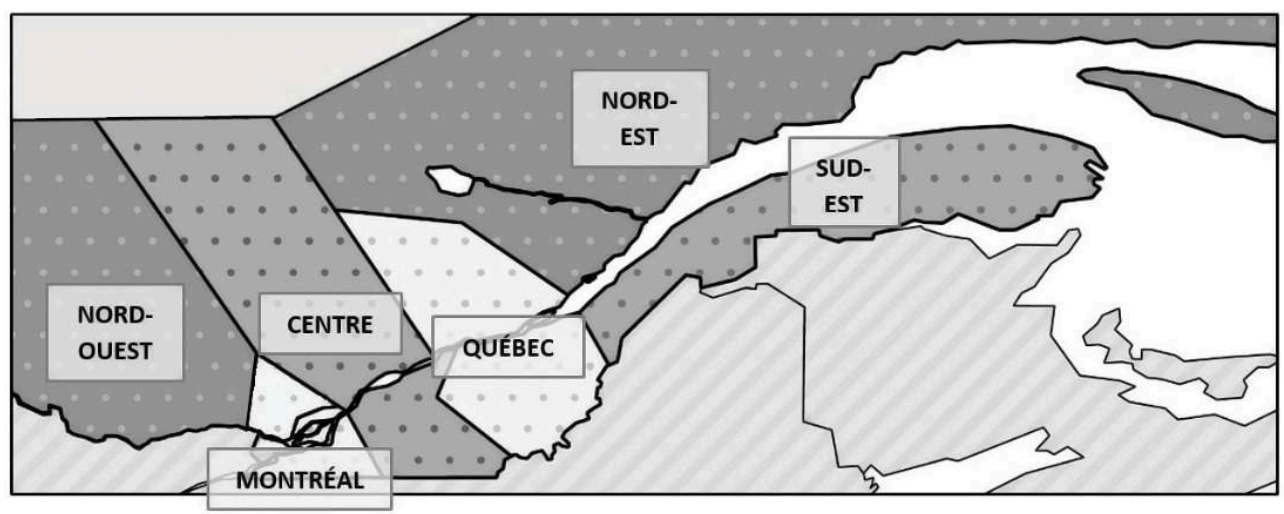

Figure 3. Carte des régions

En plus du regroupement par région, les localités du corpus sont regroupées dans une division géographique plus large. Les régions du Nord-Ouest, de Montréal et du Centre forment la zone de l'Ouest. Les régions de Québec, du Sud-Est et du Nord-Est forment la zone de l'Est. Cette séparation binaire du territoire québécois correspond à l'isoglosse traditionnelle du lieu d'articulation de /ь/ ([r] à l'Ouest, [в] à l'Est ; voir Saint-Amant Lamy, 2016).

Une distinction est également faite entre les points d'enquête de basse (moins de 4000 habitants), moyenne (entre 4000 et 40000 habitants) et haute densité (plus de 40000 habitants). 


\subsection{Traitement des données}

Les données prises en compte dans la présente étude sont les trois premiers formants des voyelles accentuées se trouvant devant un /ь/ final. Pour chaque témoin, les passages contenant une voyelle pré-rhotique ont été transcrits et segmentés grâce à l'aligneur automatique développé par Milne (2012). Les valeurs de F1, F2 et F3 à $40 \%$ de la durée de la voyelle ont été extraites à l'aide d'un script développé par Jeff Mielke ${ }^{8}$. En tout, 19198 voyelles pré-rhotiques ont été extraites. De ce nombre, 5044 ont été écartées en raison d'un environnement sonore trop bruyant, de leur durée trop courte (moins de 50 millisecondes) ou de leur statut inaccentué (prépositions (en)vers, hors, lors, par, pour, sur). Les 14154 voyelles restantes ont été normalisées selon la méthode de Bladon, Henton et Pickering (1984), qui se base sur une transformation d'une échelle en Hertz vers une échelle en Bark ${ }^{9}$. Les données aberrantes ont été exclues à partir des distances de Mahalanobis séparant chaque occurrence de la moyenne de sa catégorie, moyenne calculée dans un espace tridimensionnel $(\mathrm{F} 1 \times \mathrm{F} 2 \times \mathrm{F} 3)^{10}$. Les observations pour lesquelles on calcule une distance de Mahalanobis dépassant le troisième quartile des données par une valeur de plus de 1,5 fois l'écart interquartile ont été écartées. En tout, 13148 occurrences de toutes les voyelles pré-rhotiques ont donc été considérées valides. Pour les analyses présentées ici, seules les voyelles $/ \mathrm{e} /{ }^{11}, / 3 /, / \mathrm{D} /$ et $/ \mathrm{wD} /{ }^{12}$ ont toutefois été retenues (voir Tableau 2).

Tableau 2. Nombre d'occurrences par catégorie vocalique

\begin{tabular}{c|c|c}
\hline \hline Voyelle & $N^{\text {bre }}$ d'observations $^{\text {moyen par témoin }}$ & $\begin{array}{c}N^{\text {bre }} \mathbf{d}^{\prime} \text { occurrences } \\
\text { moyen }\end{array}$ \\
\hline \hline$/ \mathrm{e} /$ & 3441 & 22,5 \\
\hline$/ 3 /$ & 1638 & 10,7 \\
\hline$/ \mathrm{p} /$ & 616 & 4,0 \\
\hline$/ \mathrm{wD} /$ & 974 & 6,4 \\
\hline Total & 6569 & 42,9 \\
\hline \hline
\end{tabular}

\subsection{Facteurs}

Afin de bien rendre compte de la diffusion des phénomènes étudiés, les facteurs chronologiques et géographiques suivants sont considérés : l'année de naissance des témoins, leur région, leur zone et la taille de leur localité. La distribution générationnelle et géographique du corpus est résumée au Tableau 3. On constate que l'échantillon est assez bien équilibré, à l'exception des premières cohortes, où les grandes villes sont nettement sous-représentées. Ce déséquilibre est dû au parti pris méthodologique de l'ALEC en faveur d'un français québécois «traditionnel», réputé mieux conservé dans les milieux plus ruraux. Cette sous-représentation des milieux 
urbains chez les premières générations étudiées doit être prise en compte dans l'analyse des résultats.

Tableau 3. Composition de l'échantillon

\begin{tabular}{l|cccccc|c|c|c|c|c|c|c}
\hline $\begin{array}{l}\text { Date de } \\
\text { naissance }\end{array}$ & \multicolumn{9}{c|}{ Région } & \multicolumn{2}{c|}{ Zone } & \multicolumn{4}{c}{ Densité } & Total \\
\hline \hline $1872-1909$ & 5 & 6 & 6 & 7 & 9 & 14 & 17 & 30 & 37 & 10 & 0 & 47 \\
\hline $1910-1949$ & 4 & 3 & 7 & 5 & 6 & 9 & 14 & 20 & 14 & 9 & 11 & 34 \\
\hline $1950-1979$ & 5 & 6 & 7 & 4 & 7 & 8 & 18 & 19 & 7 & 13 & 17 & 37 \\
\hline $1980-1997$ & 5 & 3 & 11 & 4 & 8 & 4 & 19 & 16 & 10 & 11 & 14 & 35 \\
\hline Total & 19 & 18 & 31 & 20 & 30 & 35 & 68 & 85 & 68 & 43 & 42 & 153 \\
\hline
\end{tabular}

D'autres facteurs ont été inclus dans les analyses afin de tester certaines hypothèses émises par les grammairiens cités par Thurot (1881). Le nombre d'années d'étude des témoins a été pris en compte. Cette information n'est toutefois disponible que pour les témoins du projet PFC. La fréquence des items lexicaux par million de mots a aussi été prise en compte. Les fréquences sont tirées du Dictionnaire de fréquence des mots $d u$ français parlé au Québec (Beauchemin, Martel et Théoret, 1992). Une distinction catégorique a été faite entre les mots ayant une grande fréquence (plus de 99 occurrences par million de mots; $\mathrm{N}=9638$ ) et celle ayant une petite fréquence (moins de 100 occurrences par million de mots; $N=4516$ ). Les mots de grande fréquence sont peu nombreux. On en compte 15 pour la voyelle /e/ (affaire, arrière, bière, dernière, espère, faire, frère, grand-mère, grand-père, manière, mère, misère, père, première, rivière) et 9 pour la voyelle /wD/ (avoir, croire, falloir, histoire, noir, pouvoir, savoir, soir, voir). Chaque item lexical a été classé selon sa catégorie grammaticale. La distribution observée pour les deux variables étudiées est présentée au Tableau 4.

Tableau 4. Catégories grammaticales

\begin{tabular}{c|c|c|c|c}
\hline \hline \multirow{2}{*}{ Catégorie } & \multicolumn{2}{|c|}{ le/ } & \multicolumn{2}{|c}{ /wD/ } \\
& $\mathrm{N}$ & Ex. & $\mathrm{N}$ & \multicolumn{2}{c}{ Ex. } \\
\hline \hline Adjectif & 224 & première, laitière & 27 & noir, respiratoire \\
\hline Nom & 2347 & frère, quadrilatère & 344 & histoire, territoire \\
\hline Préposition & 19 & derrière & 0 & \\
\hline Verbe & 851 & espère, préfère & 603 & avoir, apercevoir \\
\hline Total & 3441 & & 974 & \\
\hline \hline
\end{tabular}

Enfin, puisqu'une catégorie très restreinte de mots, formée de père, mère, frère et leurs dérivés (grand-mère, compère, etc.) a fait l'objet de commentaires particuliers par certains grammairiens, celle-ci a été considérée comme dernier facteur. Les mots appartenant à cette classe lexicale représentent une partie importante des occurrences de la classe vocalique /e/ (1332 occurrences sur 3441, ou 38,7\%). 


\section{Résultats : diphtongue -oi devant /ь/}

\subsection{Aspects chronologiques}

Le timbre du noyau de la diphtongue -oi change de façon claire au cours de la période étudiée. Comme on peut le voir à la Figure 4, qui illustre la variation de F1, F2 et F3 selon l'année de naissance des témoins, c'est par une baisse marquée de F2 que ce changement se manifeste le plus explicitement. Le troisième formant connait pour sa part une hausse modérée. De leur côté, les valeurs de F1 restent relativement stables tout au long du $20^{\mathrm{e}}$ siècle.

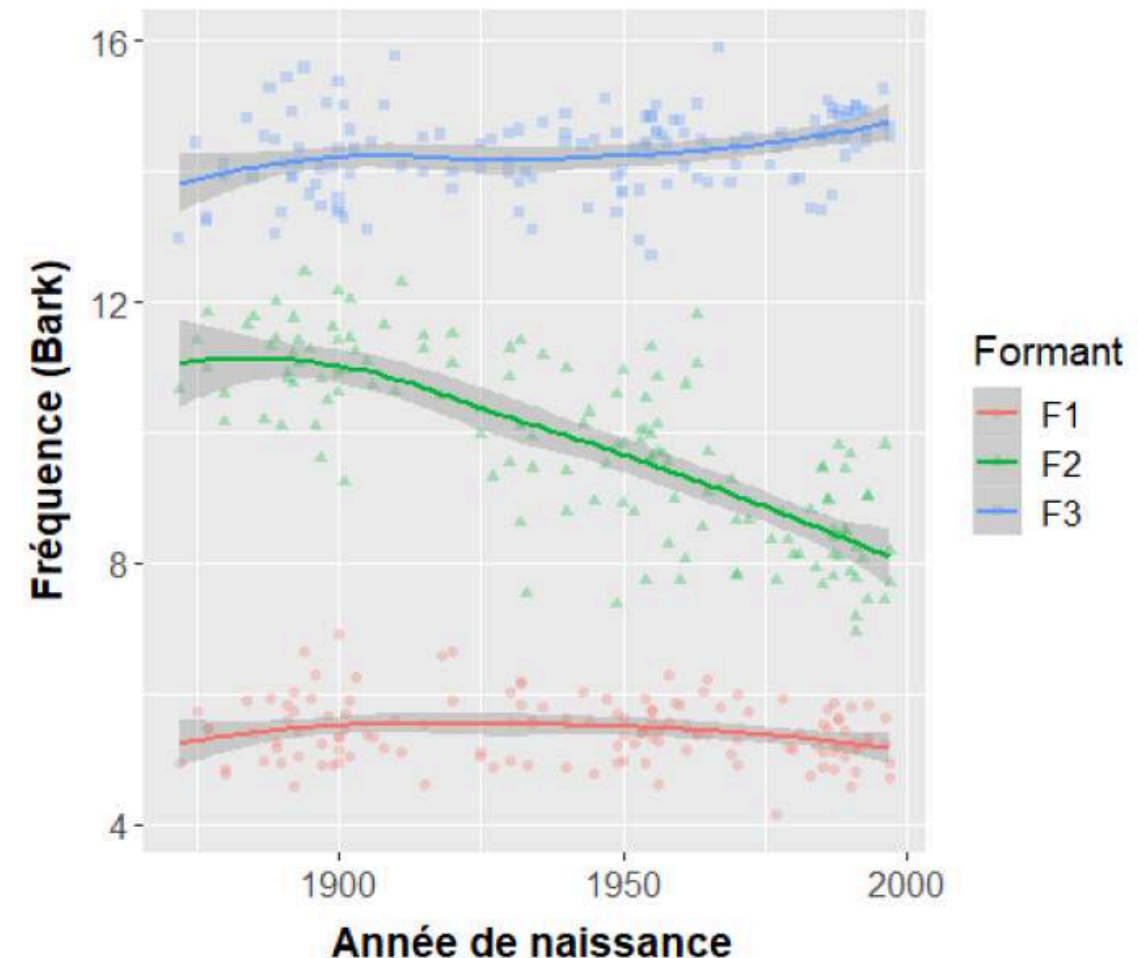

Figure 4. Évolution des fréquences de F1, F2 et F3 de la diphtongue -oi devant /b / (avec régression locale - LOESS)

Il est possible d'interpréter ces résultats comme un recul du lieu d'articulation de la diphtongue, sans variation significative de l'aperture. Ce déplacement dans l'espace vocalique est illustré à la Figure 5 . Chez la première cohorte, on constate une superposition importante entre la distribution des occurrences de la diphtongue -oi et celle de la voyelle mi-ouverture non-arrondie /3/. Chez les deux cohortes suivantes, la distribution de la diphtongue s'étend largement de l'avant à l'arrière de l'espace vocalique, chevauchant les distributions des voyelles $/ 3 /$ et $/ \mathrm{p} /$. Enfin, chez la dernière cohorte, la distribution de la diphtongue se concentre au niveau de la partie supérieure de la distribution de $/ \mathrm{p} /$. 


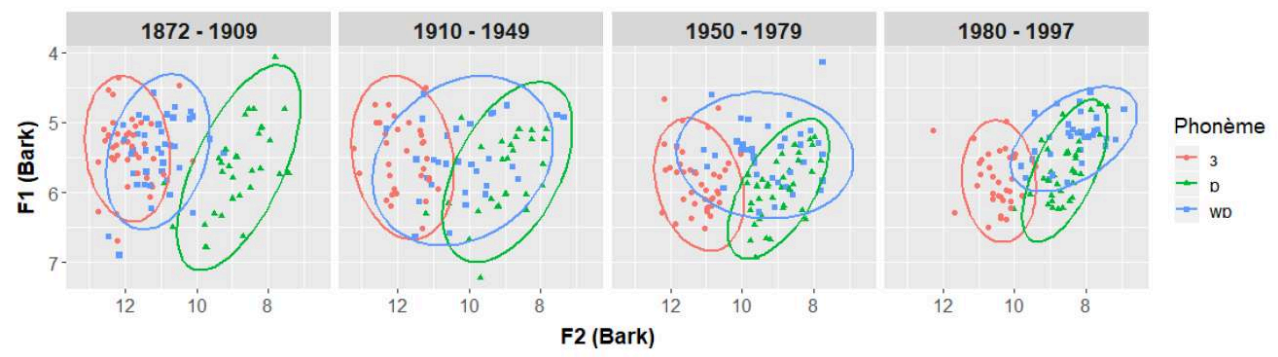

Figure 5. Valeurs moyennes individuelles de /3/, /D/ et /WD/ devant /b/ une diphtongue -oi alignée phonétiquement sur la voyelle /3/ (ex. père [рзв] - poire [рwзь]) et une diphtongue -oi alignée phonétiquement sur /o/ (еx. rime part [ррь] poire [рwDs]). Cette transition s'effectue en grande partie au cours de la période étudiée, bien que son déclenchement lui semble légèrement antérieur (voir Figure 6).

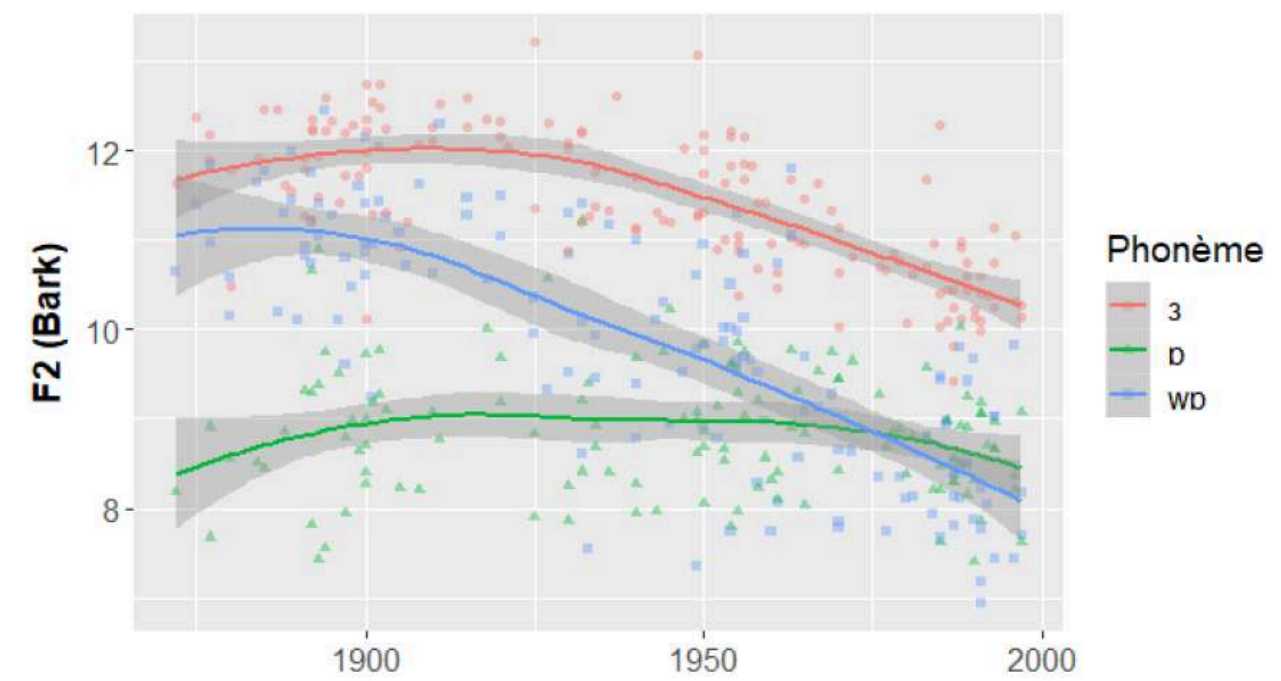

Année de naissance

Figure 6. Évolution du F2 de /3/, /D/ et /wD/ devant /b/ (avec régression locale - LOESS)

La Figure 6 pourrait suggérer un processus continu, où la cible phonétique pour la diphtongue changerait progressivement au cours $\mathrm{du} 20^{\mathrm{e}}$ siècle, d'antérieure à centrale, puis à postérieure. Cette interprétation fait toutefois fi d'un aspect important du changement. En effet, si l'on s'intéresse à la distribution des occurrences de la diphtongue, on observe une distribution bimodale (Figure 7). Or, si le changement s'était manifesté par un déplacement progressif et régulier de la cible phonétique, avec une distribution normale à chaque étape, on s'attendrait à ce que cette distribution soit unimodale. La distribution à deux pics, correspondant grosso modo aux cibles pour /3/ et $/ \mathrm{p} /$, suggère plutôt une compétition entre deux variantes phonétiquement distinctes. L'usage de la première de ces variantes, un [w3] antérieur ayant un F2 d'environ 11 Bark, aurait peu à peu diminué alors que se propageait une seconde variante [wD], postérieure et caractérisée par un F2 d'environ 8,5 Bark. Le creux entre ces deux sommets suggère l'absence de variantes ayant une cible phonétiquement intermédiaire. Il ne semble pas que la transition entre /w3/ et /wo/ se soit faite via une variante centrale de type [we] ou [wo]. 


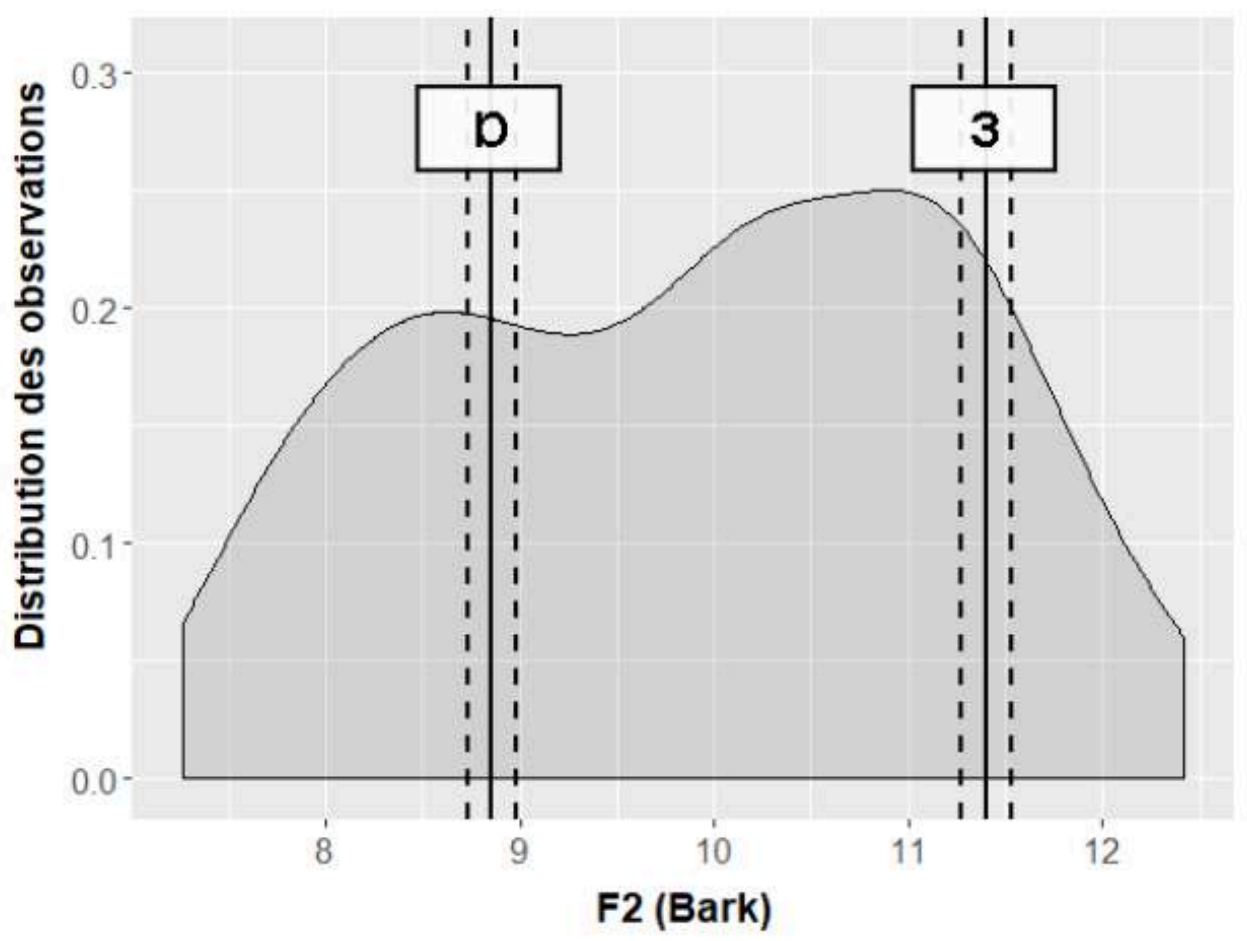

Figure 7. Distribution des observations de la diphtongue -oi selon la valeur de F2 (avec moyenne et intervalles de confiance à 95\% pour / D/ et /3/)

\subsection{Aspects géographiques}

Une régression linéaire multiple a été effectuée afin de mesurer l'effet des facteurs chronologiques et géographiques sur l'adoption des variantes postérieures de la diphtongue -oi. Les facteurs retenus sont l'année de naissance des témoins, leur zone d'origine et la taille de leur localité. La variable dépendante utilisée est la valeur de F2 moyenne pour chaque témoin. L'année de naissance et la densité de population se sont avérées significatives, mais pas la zone géographique (voir Tableau 5). Les variantes postérieures de la diphtongue sont plus répandues chez les témoins plus jeunes que chez les plus âgés. Les valeurs de F2 de la diphtongue sont également plus basses dans les grandes villes $(\sigma=8,91$; é.t. $=0,99)$ que dans les villes de tailles moyennes $(\sigma=9,68$; é.t. $=1,37 ; t(81)=2,936 ; p=0,004)$, et plus basses dans ces dernières que dans les petites localités $(\sigma=10,48$; é.t. $=1,21 ; t(98)=3,085 ; p=0,003)$. L'année de naissance s'avère toutefois être un facteur beaucoup plus prédictif que la taille de la localité, avec des coefficients de régression standardisés (Bêta) respectifs de -0,688 et -0,169. 
Tableau 5. Régression linéaire multiple - diphtongue -oi (facteurs géographiques)

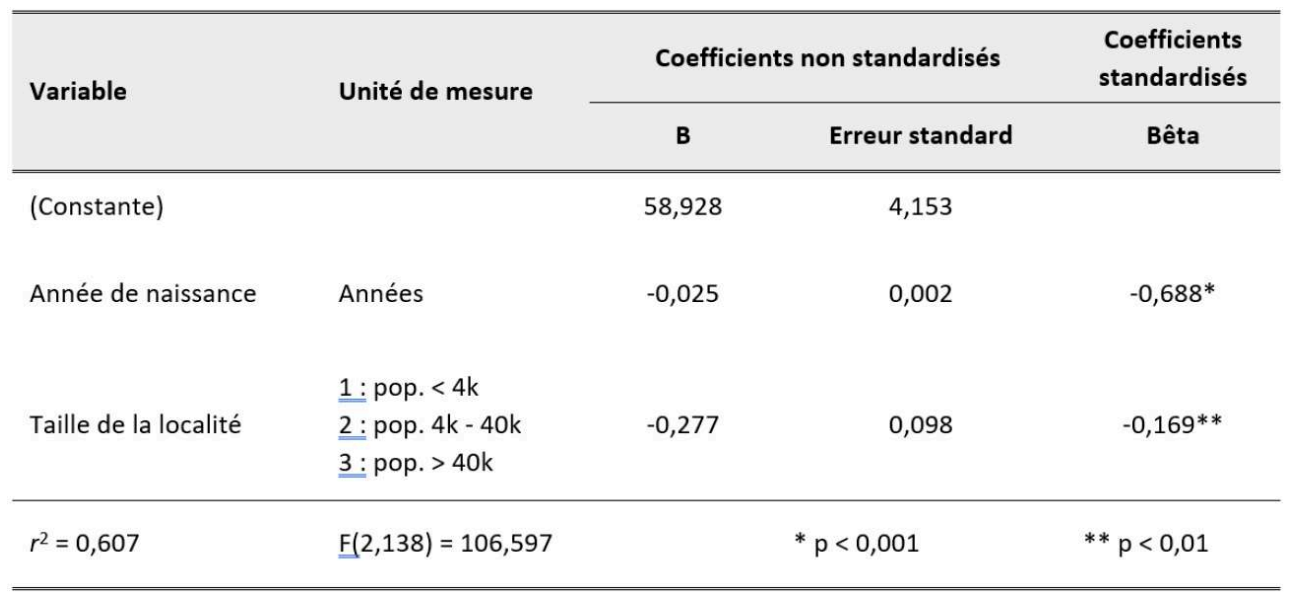

Une régression semblable à celle résumée plus haut a été effectuée en remplaçant la zone par la région d'origine, mais ce facteur ne s'est pas avéré plus significatif que le premier.

La Figure 8 montre l'évolution des valeurs de F2 de la diphtongue en fonction de la densité de population de la localité. On constate que les villes de grande taille mènent le changement, suivies par les villes de taille moyennes, suivies elles-mêmes par les plus petites localités ${ }^{13}$. Les données sont insuffisantes pour confirmer la chronologie du déclenchement $d u$ phénomène dans les grandes villes. Toutefois, si l'adoption des variantes de type $[\mathrm{wD}]$ s'est faite de façon régulière dans ces milieux, comme ce que l'on observe ailleurs, on peut faire l'hypothèse d'une adoption des nouvelles variantes vers le milieu du $19^{\mathrm{e}}$ siècle. En effet, la régression linéaire présentée plus haut prévoit, pour les grandes villes, un F2 moyen de 12 Bark (correspondant grosso modo au timbre de [3]) en 1844.

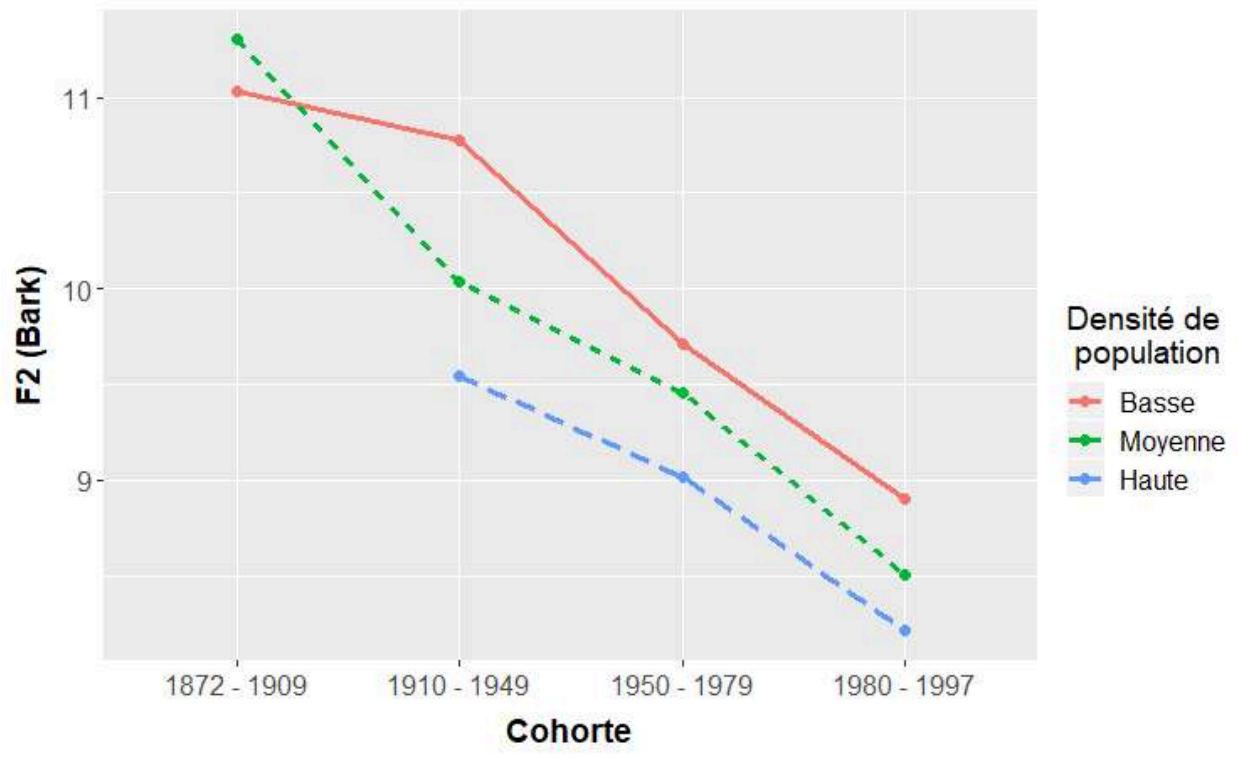

Figure 8. Effet de la taille de la localité sur les valeurs de F2 de la diphtongue -oi 


\subsection{Autres facteurs de variation}

\section{(1881) ont émis des hypothèses quant aux facteurs de variation entre [wE] et [wa]. Afin} de vérifier ces hypothèses, un certain nombre de facteurs ont été testés : la catégorie grammaticale et la fréquence des items lexicaux, ainsi que le niveau d'éducation des témoins.

Une régression linéaire a été effectuée afin de mesurer l'effet de ces facteurs, en combinaison avec ceux présentés à la section $4.2^{14}$. L'année de naissance, la taille de la localité et le niveau de scolarité ont un poids significatif dans le modèle obtenu, mais pas la fréquence lexicale, ni la catégorie grammaticale (voir Tableau 6). En plus des schémas évoqués plus haut pour l'année de naissance et la taille de la localité, il semble que les témoins plus instruits produisent des réalisations plus postérieures de la diphtongue -oi que les témoins moins scolarisés. Les facteurs de l'année de naissance (Bêta $=-0,417)$ et la taille de la localité (Bêta $=-0,202)$ sont toutefois plus prédictifs des valeurs de F2 de la diphtongue que le nombre d'années d'études (Bêta $=-0,126$ ).

Tableau 6. Régression linéaire multiple - diphtongue -oi (autres facteurs)

\begin{tabular}{|c|c|c|c|c|}
\hline \multirow{2}{*}{ Variable } & \multirow{2}{*}{ Unité de mesure } & \multicolumn{2}{|c|}{ Coefficients non standardisés } & \multirow{2}{*}{$\begin{array}{c}\begin{array}{c}\text { Coefficients } \\
\text { standardisés }\end{array} \\
\text { Bêta }\end{array}$} \\
\hline & & B & Erreur standard & \\
\hline (Constante) & & 64,619 & 4,234 & \\
\hline Année de naissance & Années & $-0,027$ & 0,002 & $-0,417$ \\
\hline Taille de la localité & $\begin{array}{l}1: \text { pop. }<4 \mathrm{k} \\
\text { 2: pop. } 4 \mathrm{k}-40 \mathrm{k} \\
\underline{\underline{3:} \text { pop. }>40 \mathrm{k}}\end{array}$ & $-0,348$ & 0,058 & $-0,202$ \\
\hline Années d'éducation & Années & $-0,065$ & 0,017 & $-0,126$ \\
\hline$r^{2}=0,246$ & $\underline{\underline{F}}(3,707)=76,724$ & & & ${ }^{*} \mathrm{p}<0,001$ \\
\hline
\end{tabular}

La Figure 9, en illustrant l'interaction entre les facteurs de la régression présentée plus haut, nous renseigne plus précisément sur la diffusion des variantes postérieures de la diphtongue -oi selon le degré d'instruction ${ }^{15}$. Chez la cohorte 1910 - 1949, les témoins plus éduqués tendent à produire des variantes plus proches de [wD] (valeurs de F2 plus basses) que les témoins moins instruits, et ce peu importe la taille de la localité. Cette tendance semble toutefois s'estomper dans les grandes villes chez la cohorte 1950 1979, où le niveau d'instruction ne semble plus avoir d'effet sur les réalisations de la diphtongue. Ceci pourrait indiquer que le changement y est en grande partie complété. Enfin, chez la cohorte 1980 - 1979, la tendance au maintien des variantes de type [w3] parmi les témoins les moins scolarisés ne s'observe plus que dans les plus petites localités ${ }^{16}$. 


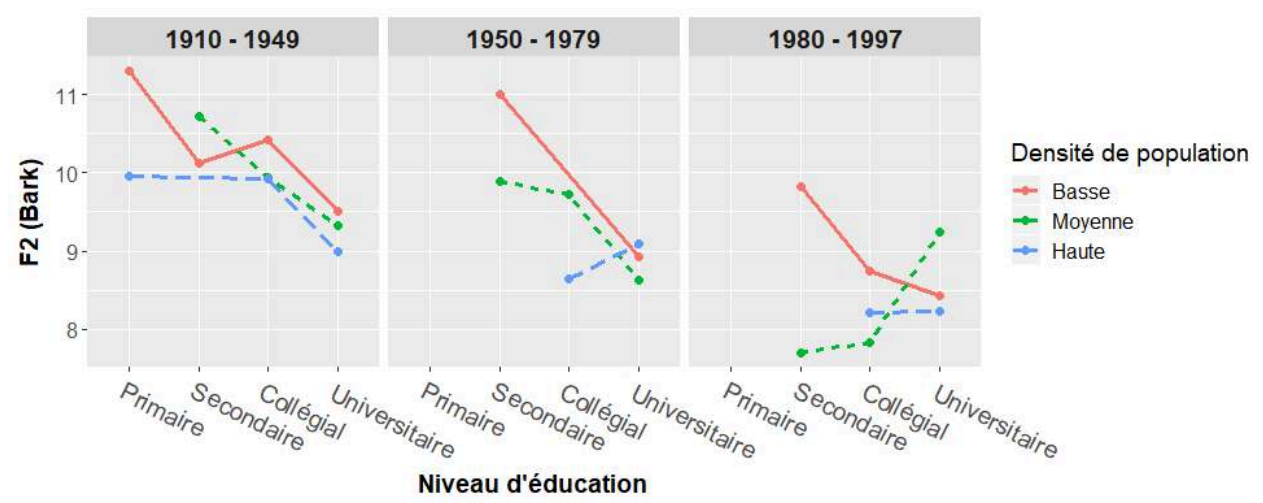

Figure 9. Effet de la taille de la localité et du nombre d'années d'étude sur les valeurs de F2 de la diphtongue -oi (avec droites de régression)

\section{Résultats : neutralisation de /е/ - /з/ devant /ь/}

\subsection{Aspects chronologiques}

On observe, au cours du $20^{\mathrm{e}}$ siècle, un phénomène général d'ouverture et de rétraction des voyelles pré-rhotiques du français laurentien (Saint-Amant Lamy, 2020). Les voyelles /e/ et /3/ n'échappent pas à ce processus, comme le démontrent la montée de $\mathrm{F} 1$ et la chute de F2 illustrées à la Figure 10. C'est en parallèle à ce phénomène que s'opère la neutralisation entre les deux phonèmes, neutralisation par laquelle les voyelles historiquement prononcées [e] prennent le timbre [3]. La différence d'aperture individuelle moyenne (manifestée par la différence de F1) entre les deux voyelles s'amenuise tout au long de la période étudiée.
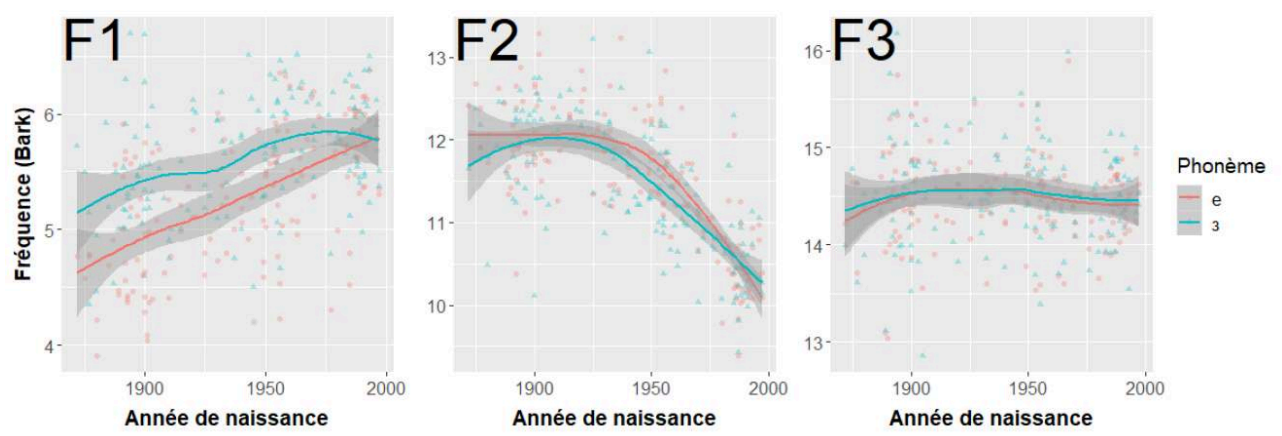

Figure 10. Évolution des valeurs de F1, F2 et F3 pour /e/ et /3/ devant /b / (avec régression locale LOESS)

La neutralisation progressive des deux voyelles est rendue explicite à la Figure 11. Les distributions de /e/ et / $3 /$ dans un espace F1×F2 se chevauchent de façon importante chez les trois premières cohortes, mais demeurent distinctes. La superposition est toutefois complète chez la cohorte 1980 - 1997, où le contraste est perdu. 


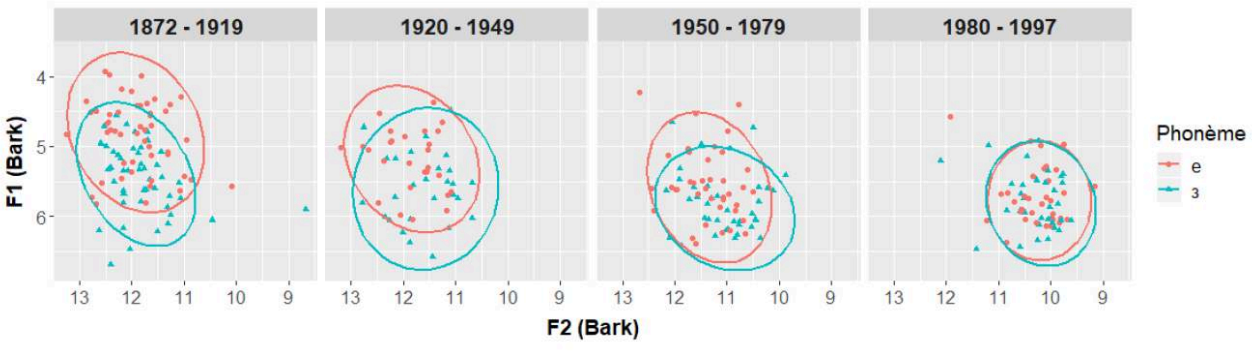

Figure 11. Valeurs moyennes individuelles de /e/ et /3/ devant / $\mathbf{b} /$

Pour chaque témoin du corpus, un indice de neutralisation est mesuré en soustrayant le F1 moyen de la catégorie /e/ du F1 moyen de la catégorie /3/. On constate ainsi une tendance à la baisse de cet indice sur toute la période étudiée, ce qui suggère que la neutralisation est déjà en marche chez les locuteurs nés à la fin du $19^{\mathrm{e}}$ siècle. Elle est complétée (différence de fréquence de zéro) chez ceux nés à la toute fin du $20^{\circ}$ siècle (voir Figure 12).

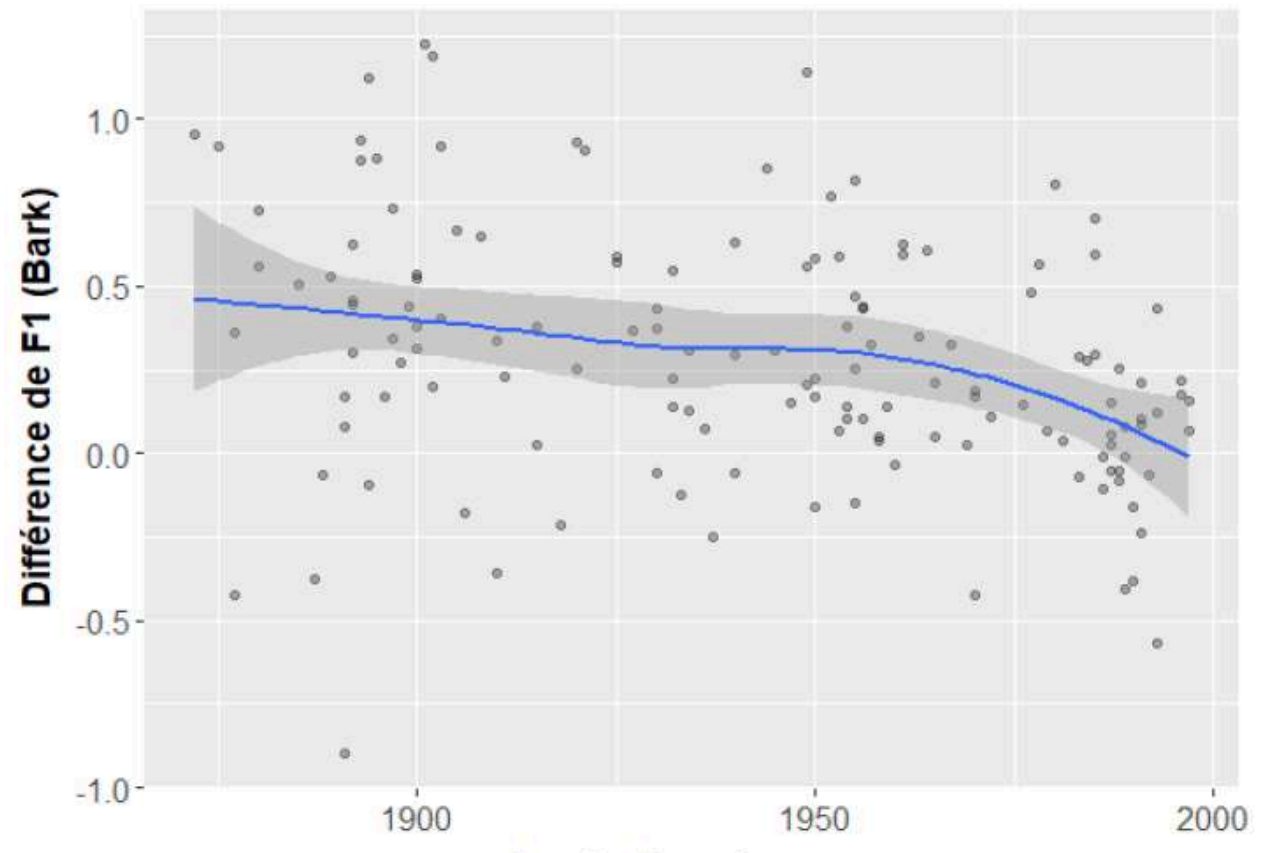

Année de naissance

Figure 12. Différence individuelle moyenne de F1 entre /3/ et /e/ (avec régression locale - LOESS)

La tendance illustrée à la Figure 12 et basée sur des moyennes individuelles occulte toutefois un aspect crucial du phénomène de neutralisation. La trajectoire continue pourrait suggérer un déplacement graduel des réalisations des voyelles de la catégorie /e/ dans l'espace vocalique. La distribution des occurrences selon leur valeur de F1 contredit toutefois cette hypothèse. Alors qu'un déplacement phonétiquement progressif devrait produire une distribution unimodale, on constate plutôt une bimodalité des observations (voir Figure 13). Ceci suggère que les anciennes réalisations de type [e] (ayant une cible de F1 d'environ 4,9 Bark) ont progressivement été supplantées par des réalisations de type [3] (ayant une cible de F1 d'environ 5,6 Bark), alignées phonétiquement sur les réalisations du phonème $/ 3 /$. 


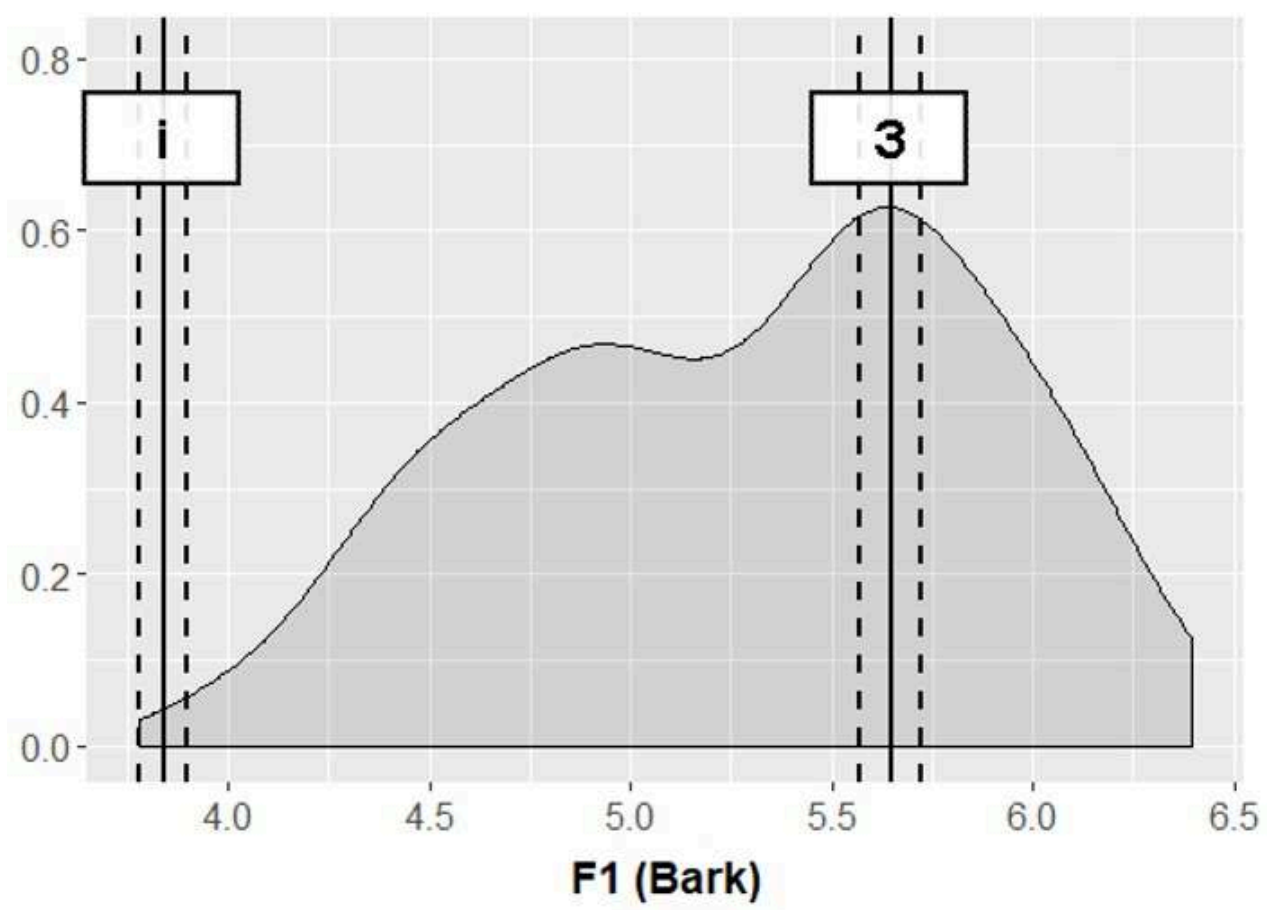

Figure 13. Distribution des observations de /e/ selon la valeur de F1 (avec moyenne et intervalles de confiance à $95 \%$ pour /i/ et /3/)

\subsection{Aspects géographiques}

Afin de mesurer l'effet des facteurs chronologiques et géographiques sur la neutralisation de /e/-/3/, une régression linéaire a été effectuée. Les facteurs retenus sont l'année de naissance des témoins, la zone et la taille de la localité. La variable dépendante est la différence individuelle moyenne de F1 entre les occurrences de la catégorie /e/ et celles de la catégorie $/ 3 /$. Tous les facteurs se révèlent significatifs dans le modèle obtenu (voir Tableau 7). Le contraste entre /e/ et /3/ se maintient le mieux chez les locuteurs âgés que chez les plus jeunes. On constate aussi qu'il est plus marqué à l'Est qu'à l'Ouest. L'opposition s'avère plus faible dans les grandes villes $(\sigma=0,133$; é.t. $=0,275)$ que dans les villes de tailles moyennes $(\sigma=0,335$; é.t. $=0,340 ; \mathrm{t}(82)=2,991 ; \mathrm{p}=$ $0,004)$, et que dans les petites localités $(\sigma=0,488$; é.t. $=0,527 ; \mathrm{t}(103)=4,017 ; \mathrm{p}<0,001)$. Il n'y a pas de différence significative entre les petites et moyennes localités. L'année de naissance s'avère être le facteur le plus prédictif, suivi par la zone et la taille de la ville (coefficients de régression standardisés respectifs -0,292 0,179 et -0,096). 
Tableau 7. Régression linéaire multiple - contraste /e/-/3/ (facteurs géographiques)

\begin{tabular}{|c|c|c|c|c|}
\hline \multirow{2}{*}{ Variable } & \multirow{2}{*}{ Unité de mesure } & \multicolumn{2}{|c|}{ Coefficients non standardisés } & \multirow{2}{*}{$\begin{array}{c}\begin{array}{c}\text { Coefficients } \\
\text { standardisés }\end{array} \\
\text { Bêta }\end{array}$} \\
\hline & & B & Erreur standard & \\
\hline (Constante) & & 6,213 & 1,771 & \\
\hline Année de naissance & Années & $-0,003$ & 0,001 & $-0,292^{*}$ \\
\hline Zone & $\begin{array}{l}1: \text { Ouest } \\
2: \text { Est }\end{array}$ & 0,141 & 0,062 & $0,179 * *$ \\
\hline Taille de la localité & $\begin{array}{l}\underline{1: \text { pop. }<4 \mathrm{k}} \\
\underline{2: \text { pop. } 4 \mathrm{k}-40 \mathrm{k}} \\
\underline{\underline{3:} \text { pop. }>40 \mathrm{k}}\end{array}$ & $-0,045$ & 0,041 & $-0,096^{* *}$ \\
\hline$r^{2}=0,176$ & $\underline{\underline{F}(3,143)}=9,954$ & & & $\begin{array}{ll}* & p<0,001 \\
* * & p<0,05\end{array}$ \\
\hline
\end{tabular}

Comme on peut le voir à la Figure 14 , il semble que la neutralisation de /e/ et $/ 3 /$ soit la plus avancée dans les grandes villes, toutes zones confondues, et la moins avancée dans les petites localités de l'Est. De façon générale, l'effet de la taille de la localité est plus saillant à l'Est. Puisque nous n'avons vraisemblablement accès qu'aux dernières étapes du phénomène à l'Ouest, il est difficile d'en extrapoler la trajectoire avant la période étudiée. Considérant une distance originale de 0,6 Bark entre /e/ et /3/ (soit la distance observée chez les plus vieux témoins ruraux de l'Est), la régression présentée plus haut prévoit une date approximative pour le début du changement dans la seconde moitié du $19^{e}$ siècle (1873).

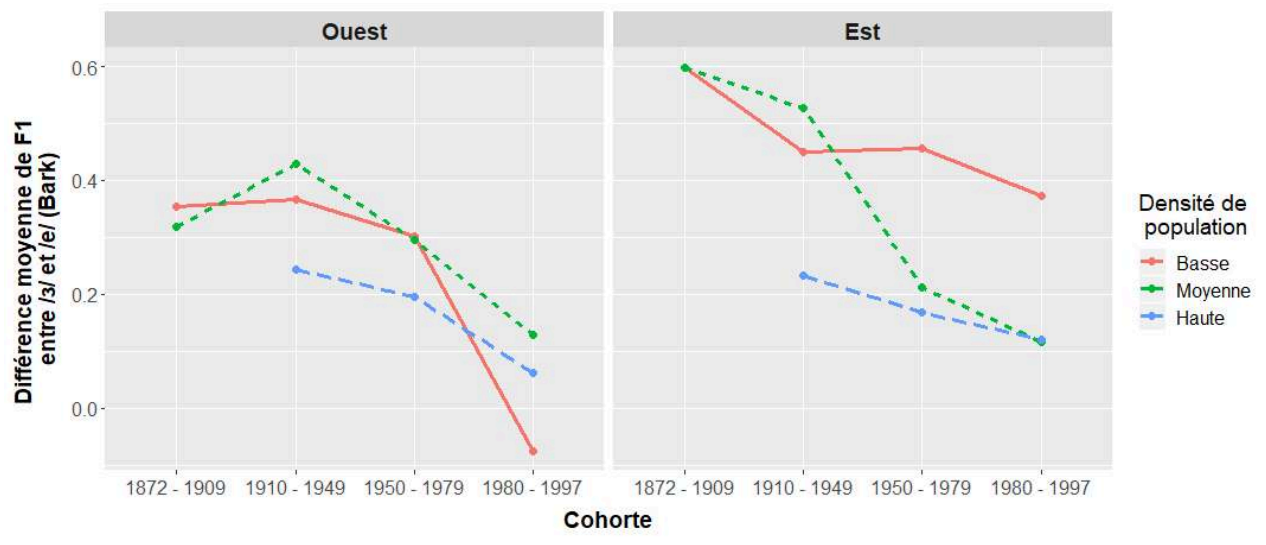

Figure 14. Effet de la taille de la localité et de la zone sur la différence /e/-/3/ (F1)

41 La régression effectuée plus haut a été reprise, en remplaçant le facteur de la zone par le facteur de la région. Le modèle obtenu $\left(r^{2}=0,175 ; \mathrm{F}(7,139)=4,207 ; \mathrm{p}<0,001\right)$ permet de d'ordonner les différentes régions selon leur coefficient de régression, et donc leur taux de neutralisation respectif. Des coefficients plus élevés sont associés à une plus grande différence de $\mathrm{F} 1$ entre /e/ et /3/, et donc à des taux de neutralisation plus bas. Les résultats sont présentés au Tableau 8 . On constate que la neutralisation des voyelles moyennes devant $/ \mathrm{B} /$ est la plus avancée dans les régions de Montréal et du Centre, suivies du Nord-Ouest, puis des régions du Nord-Est de Québec et, enfin, du Sud-Est. 


\begin{tabular}{c|c|c}
\hline \hline \multirow{2}{*}{ Zone } & Région & $\begin{array}{c}\text { Coefficient de } \\
\text { régression }\end{array}$ \\
\hline \hline \multirow{4}{*}{ Ouest } & Montréal & $-0,246$ \\
\cline { 2 - 3 } & Centre & $-0,217$ \\
\cline { 2 - 3 } & Nord-Ouest & $-0,205$ \\
\hline \multirow{4}{*}{ Est } & Nord-Est & $-0,175$ \\
\cline { 2 - 3 } & Québec & $-0,138$ \\
\cline { 2 - 3 } & Sud-Est & 0,000 \\
\hline \hline
\end{tabular}

\subsection{Autres facteurs de variation}

Une seconde régression linéaire a été effectuée afin de tester, entre autres, certaines des hypothèses rapportées par Thurot (1881). Aux facteurs chronologiques et géographiques jugés significatifs précédemment (année de naissance, zone, taille de la ville), les facteurs suivants ont été ajoutés : la présence d'un / $\mathrm{j}$ / avant la voyelle (mots en -ière), la fréquence lexicale, la classe lexicale père/mère/frère, la catégorie grammaticale et le nombre d'années d'éducation des témoins. La variable dépendante retenue est la différence entre la moyenne individuelle de F1 pour la catégorie /3/ et le F1 de chacune des occurrences de /e/. Tous les facteurs s'avèrent significatifs dans le modèle obtenu (voir Tableau 9 et Figure 15). On constate que des niveaux élevés d'éducation, les mots moins fréquents et la catégorie père/mère/frère sont associés à une plus grande neutralisation du contraste /e/-/3/. À l'inverse, il semble que la présence $\mathrm{de} / \mathrm{j} /$ avant la voyelle et la catégorie adjectivale correspondent à un meilleur maintien de l'opposition phonémique. Ce dernier facteur (Bêta $=0,045$ ) est toutefois moins prédictif que les autres (Bêta $>0,103$ ). 
Tableau 9. Régression linéaire multiple - contraste /e/-/3/ (tous facteurs)

\begin{tabular}{|c|c|c|c|c|}
\hline \multirow{2}{*}{ Variable } & \multirow{2}{*}{ Unité de mesure } & \multicolumn{2}{|c|}{ Coefficients non standardisés } & \multirow{2}{*}{$\begin{array}{c}\begin{array}{c}\text { Coefficients } \\
\text { standardisés }\end{array} \\
\text { Bêta }\end{array}$} \\
\hline & & B & Erreur standard & \\
\hline (Constante) & & 13,271 & 1,088 & \\
\hline Année de naissance & Années & $-0,006$ & 0,001 & $-0,208^{*}$ \\
\hline Zone & $\begin{array}{l}1: \text { Ouest } \\
2: \text { Est }\end{array}$ & 0,221 & 0,023 & $0,175^{*}$ \\
\hline Années d'éducation & Années & $-0,032$ & 0,004 & $-0,146^{*}$ \\
\hline Fréquence lexicale & $\begin{array}{l}1:+ \text { de } 99 / \text { million } \\
2:- \text { de } 100 / \text { million }\end{array}$ & $-0,235$ & 0,040 & $-0,123^{*}$ \\
\hline Père, mère, frère, etc. & $\begin{array}{l}1: \text { père, mère, frère, etc. } \\
0: \text { autres }\end{array}$ & $-0,155$ & 0,026 & $-0,121^{*}$ \\
\hline /j/ avant voyelle (-ière) & $\begin{array}{l}1: \text {-ière } \\
0 \text { :-ère }\end{array}$ & 0,151 & 0,036 & $0,103^{*}$ \\
\hline Densité de population & $\begin{array}{l}1: \text { pop. }<4 k \\
2: \text { pop. } 4 k-40 k \\
3: \text { pop. }>40 k\end{array}$ & $-0,067$ & 0,015 & $-0,083^{*}$ \\
\hline Adjectif & $\begin{array}{l}1: \text { adjectif } \\
0: \text { autres }\end{array}$ & 0,117 & 0,052 & $0,045^{* *}$ \\
\hline$r^{2}=0,155$ & $F(8,2686)=61,559$ & & & $\begin{array}{ll}* & \mathrm{p}<0,001 \\
* * & \mathrm{p}<0,05\end{array}$ \\
\hline
\end{tabular}
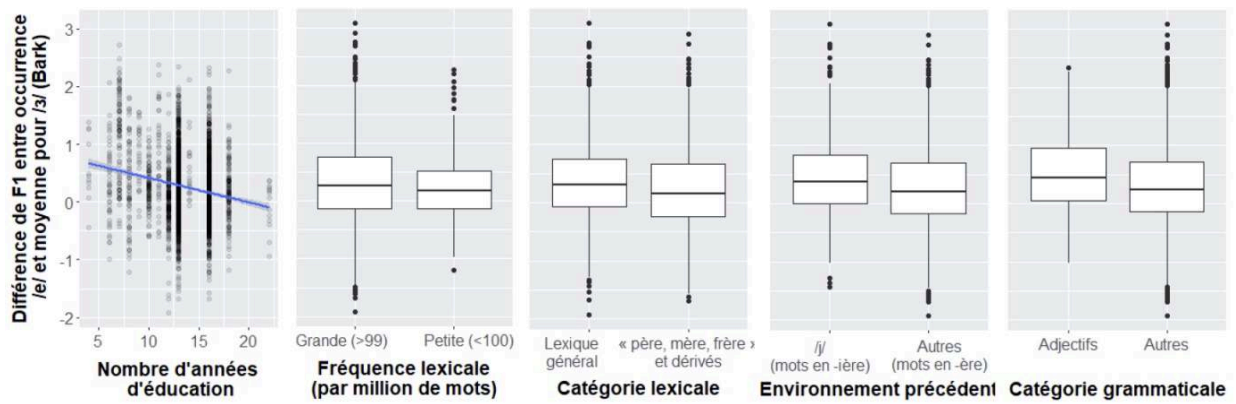

Figure 15. Effet des facteurs significatifs (régression - différence de F1 entre /e/-/3/)

\section{Analyse}

Les deux traits conservateurs étudiés, soit la variante [w3] de la diphtongue -oi et le maintien du /е/ devant /в/ final, connaissent un déclin important au cours de la période étudiée.

La diphtongue, essentiellement antérieure à la fin du $19^{\mathrm{e}}$ siècle, est complètement postérieure à la fin du $20^{\mathrm{e}}$ siècle. Le timbre moyen de la diphtongue au début de la période étudiée n'est toutefois déjà plus tout à fait aligné sur la voyelle $/ 3 /$. On peut donc déduire que le changement était déjà entamé. Il l'était certainement dans les grandes villes, bien que les données disponibles ne permettent pas de l'observer directement. Si le rythme de changement observé à partir de 1872 était le même lors de la période qui précède, une apparition de la variante [wo] en milieu urbain au milieu du $19^{\mathrm{e}}$ siècle est vraisemblable. Le changement de cible phonétique pour la diphtongue 
s'opère de façon catégorique, avec un déclin progressif des occurrences de type [w3] face à l'adoption d'une nouvelle cible [wD].

Le déclin du /e/ pré-rhotique, déjà en cours chez les témoins nés à la fin du $19^{\mathrm{e}}$ siècle, se conclut à la fin du $20^{\mathrm{e}}$ siècle, où la neutralisation avec /3/ est complétée. Puisque le changement est déjà entamé dans les petites localités de l'Ouest chez la cohorte 1872 1909, il est vraisemblable qu'il ait déjà été présent dans les grandes villes de cette zone chez la génération précédente, celle née au milieu $d u 19^{\mathrm{e}}$ siècle. Le phénomène semble s'être produit de façon catégorique, par l'abandon des réalisations [e] et l'adoption des réalisations [3], et non par un déplacement progressif de la cible phonétique dans l'espace vocalique.

\subsection{Une transmission au $18^{\mathrm{e}}$ siècle?}

Les résultats obtenus ne permettent pas de conclure que le déclin de [w3] et de [e] devant /в/ ait eu le temps de se propager au Québec avant la fin du Régime français. Les deux variantes conservatrices y sont encore très fortement majoritaires à la fin du $19^{\mathrm{e}}$ siècle. Si les nouvelles variantes de prestige [wD] et [3] avaient eu le temps de s'imposer au $18^{\mathrm{e}}$ siècle dans la vallée du Saint-Laurent, on pourrait s'attendre à ce que celles-ci se soient diffusées de façon notable.

C'est ce qui se serait produit notamment avec la consonne rhotique. Morin (2002) avance l'idée que le $[\mathrm{H}]$ dorsal aurait été introduit dans la ville de Québec entre la fin du $17^{\mathrm{e}}$ siècle et la fin du Régime français, par des vagues successives d'administrateurs et de colons urbains emportant avec eux les nouvelles normes de prononciation métropolitaines. La nouvelle variante de /ь/ n'aurait pas eu le temps de se diffuser au reste du territoire québécois, où le $[\mathrm{r}]$ apical était en usage, avant la Conquête de 1763. La nouvelle variante $[\mathrm{B}]$ se serait diffusée à l'Est du Québec à partir de la Vieille Capitale, tandis que l'Ouest aurait conservé son [r], désormais caractéristique de la ville de Montréal.

Un schéma comparable ne s'observe pas pour la réalisation de la diphtongue -oi ou l'opposition /e/-/3/ devant /ь/. La zone Est ou la région de Québec ne font aucunement figure de précurseures dans l'adoption des nouvelles formes. Les administrateurs français du $18^{\mathrm{e}}$ siècle ne semblent donc pas avoir transporté ces innovations au Canada. Du moins, s'ils l'ont fait, celles-ci ne se sont pas implantées durablement après leur départ.

Dans le cas de l'opposition /e/-/3/, on trouve bien un schéma de diffusion géographique, mais celui-ci a comme foyer de propagation la ville de Montréal. Notons que la population de cette dernière ne dépasse celle de Québec que dans la décennie 1830 (Lamonde, 2000: 402). Elle devient donc probablement le principal creuset d'innovation et de diffusion linguistique du Canada français vers le milieu du $19^{\mathrm{e}}$ siècle. C'est vraisemblablement à cette époque qu'il faut chercher l'origine québécoise des phénomènes étudiés, et non dans la période qui précède la Conquête de 1763.

Certains schémas de variation observés viennent toutefois jeter une ombre sur $l^{\prime}$ hypothèse d'un déclin tardif ( $19^{\mathrm{e}}$ siècle) de [e] devant $/ \mathrm{L} /$. Au Québec, on note un plus grand maintien du [e] dans les séquences -ière et dans les adjectifs que dans les séquences -ère ou les noms/prépositions/verbes, ce qui correspond à la variation rapportée en France aux $16^{\mathrm{e}}$ et $17^{\mathrm{e}}$ siècles (voir section 2.1). On peut supposer que, dans la France du $19^{e}$ siècle, ces cas de variation s'étaient déjà résolus en faveur de la 
variante mi-ouverte. L'alternance observée au Québec serait-elle alors la manifestation d'une transmission plus hâtive? La possibilité ne peut être écartée d'emblée. D'autres pistes d'explication que la transmission transatlantique peuvent toutefois être explorées. Par exemple, le maintien du [e] pourrait être attribuable à la coarticulation avec [j] dans les mots en -ière, le [e] des adjectifs féminins pourrait être analogique avec

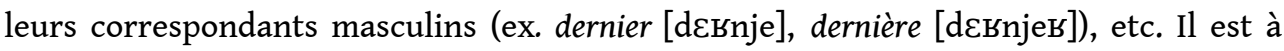
noter que les mots de la catégorie mère/père/frère sont caractérisés au Québec par un usage plus grand de la variante mi-ouverte, contrairement à ce qui est observé par les auteurs cités par Thurot (1881). On ne remarque pas non plus, comme il a été rapporté, un son intermédiaire entre la voyelle mi-fermée et la mi-ouverte.

51 Le cas de la diphtongue -oi est encore plus clair quant à l'absence de correspondance entre la variation française et québécoise. On n'observe pas une cible distincte qui aurait comme réalisation un "son intermédiaire entre $a$ et $\grave{e}$ ». Il ne semble pas non plus, comme il a été suggéré, que la catégorie grammaticale conditionne l'abandon de la variante [w3]. A priori, la présence de certains cas de variation analogues en France et au Québec ne semble pas être un argument suffisamment robuste pour infirmer l'hypothèse d'un déclin tardif de [w3] et [e].

\subsection{Une transmission au $19^{\mathrm{e}}$ siècle}

Le déclin des [w3] et [e] pré-rhotiques dans les grandes villes du Québec précède d'environ une génération celui observé dans les plus petites localités. Comme les deux changements sont déjà entamés chez les locuteurs ruraux nés à partir de 1872, on peut faire l'hypothèse qu'ils prennent leur origine dans les milieux urbains, vers la moitié du $19^{\mathrm{e}}$ siècle.

Dans les deux cas, la diffusion des nouvelles formes est conditionnée par la taille de la localité et le niveau d'éducation des témoins. Ce schéma de propagation hiérarchique est caractéristique de l'adoption consciente de nouvelles formes linguistiques prestigieuses. Les formes [wD] et [3] auraient d'abord gagné la faveur chez l'élite intellectuelle des grandes villes. Ces locuteurs auraient été en contact avec les nouvelles normes de prononciation française, soit de façon indirecte, avec des ouvrages comme Maguire (1841), soit de façon directe, par la fréquentation d'immigrants français installés au Québec à partir de la décennie $1840^{17}$. Les voyages que l'intelligentsia québécoise effectue à partir du Second Empire ont également pu jouer un rôle dans le relai des normes de prononciations européennes (voir Morin, 2002). Les nouvelles variantes se seraient ensuite graduellement propagées aux locuteurs moins scolarisés et dans les plus petits milieux, où le contact avec la norme française était jusqu'alors très limité.

54 En plus de la taille des localités, le déclin du [e] pré-rhotique est conditionné par la position géographique de celles-ci. La neutralisation commence dans la région de Montréal, avant de gagner les régions limitrophes (Nord-Ouest et Centre), puis les régions du Nord-Est, de Québec, et du Sud-Est. Ce type de diffusion hybride (modèle de la gravité ; Trudgill, 1974) pourrait correspondre à une situation où le statut des nouvelles formes de prestige est ambigu, et où les interactions interpersonnelles jouent un rôle clé dans la diffusion. Cette interprétation est compatible avec le discours normatif présent au $19^{\mathrm{e}}$ siècle. Alors que la réalisation [w3] de la diphtongue est explicitement condamnée, le [e] pré-rhotique ne l'est que tacitement. La présence de 
prescriptions écrites et imprimées aurait permis à la variante [wo] de s'implanter relativement rapidement sur tout le territoire québécois, possiblement via le réseau scolaire. La perte de l'opposition /e/-/3/, de son côté, aurait d'abord atteint les locuteurs éduqués et urbains en contact avec la nouvelle norme française mais, en l'absence de condamnations normatives largement diffusées, elle se serait propagée plus lentement au reste de la population. Elle aurait ruisselé, au fil des échanges interpersonnels, aux classes moins éduquées de la région montréalaise et aux villages environnants, avant de se propager plus loin vers l'Est, d'abord dans les villes, où les réseaux intellectuels de l'Ouest auraient eu des antennes, puis aux milieux plus ruraux.

Ce processus de rattrapage normatif par rapport à la France, observé à partir du milieu du $19^{e}$ siècle au Québec, ne se limite vraisemblablement pas aux deux phénomènes étudiés ici. Par exemple, Kemp et Yaeger-Dror (1991) suggèrent que l'abandon en français québécois $\mathrm{du}[\mathrm{a}]$ postérieur dans le suffixe -ation pourrait être le produit d'un alignement sur la norme française du $19^{\mathrm{e}}$ siècle (où l'on trouve la prononciation [asjõ]). Cette hypothèse est soutenue par le discours normatif de l'époque. Par exemple, dans son manuel, Maguire informe son lectorat québécois que l'Académie française ne reconnaît qu'une distinction de longueur, et non de timbre, entre les deux « $a$ » (1841: 101). Il transcrit ainsi le «a » de cognation, équitation, imprégnation et stagnation de la même façon que celui de balai ou Madrid, soit par une voyelle basse antérieure.

\section{Conclusion}

Le déclin des $[w \varepsilon]$ et $[e]$ pré-rhotiques en France est survenu trop tôt pour être adéquatement analysé à l'aide des outils acoustiques modernes. La survivance de ces traits en français québécois rend possible une telle analyse et, par le fait même, permet de mieux comprendre ce moment de transition entre deux états du système sonore du français. Nous avons vu qu'en seulement quelques générations, les variantes anciennes sont abandonnées, et remplacées par les variantes nouvelles. Ce changement s'opère de façon catégorique, sans l'adoption de cibles phonétiquement intermédiaires. Les innovations, associées à la langue de prestige, apparaissent d'abord chez les locuteurs instruits des grandes villes avant de se propager au reste de la population.

Cette étude met également en lumière la relation normative complexe qui existe entre la France et le Québec. Sous le Régime français, la colonie est en contact avec l'évolution des normes linguistiques métropolitaines, mais pas de façon absolue. Les changements au niveau de la diphtongue -oi et de l'opposition /e/-/3/, rapportés par les auteurs français, ne traversent pas l'Atlantique avant la Conquête 1763. Il faut attendre le milieu du $19^{\mathrm{e}}$ siècle, avec le rétablissement des contacts France-Québec et la naissance d'un véritable discours normatif canadien-français pour que la norme française soit de nouveau relayée en Amérique. Les intellectuels et le clergé jouent vraisemblablement un rôle central dans ce processus.

Il serait intéressant d'approfondir la question de l'évolution des liens linguistiques (normes et usages) existant entre la France et le Québec en comparant les productions linguistiques de locuteurs québécois nés à la fin du $19^{\mathrm{e}}$ siècle avec celles de leurs contemporains français. Une telle entreprise permettrait de mieux modéliser l'histoire des mécanismes géolinguistiques de la francophonie et d'entrevoir les réalités linguistiques que peuvent occulter les discours sur la norme. 


\section{BIBLIOGRAPHIE}

BEAUCHEMIN, N., MARTEL, P. \& THÉORET, M., 1992, Dictionnaire de fréquence des mots du français parlé au Québec : fréquence, dispersion, usage, écart réduit, Berne, Peter Lang.

BIBAUD, M., 1842, L'Encyclopédie canadienne : journal historique, littéraire et scientifique, Montréal, M. Bibaud.

BLADON, R. A., HENTON, C. G., \& PICKERING, J., 1984, « Towards an auditory theory of speaker normalization », Language \& Communication, vol. $4, n^{\circ} 1$, p. 59-69.

BOUCHARD, C., 2012, Méchante langue : la légitimité linguistique du français parlé au Québec, Montréal, Presses de l'Université de Montréal.

CÔTÉ, M.-H., 2014, « Le projet PFC et la géophonologie du français laurentien », dans J. Durand, G. Kristoffersen, B. Laks et J. Peuvergne (éds.), La phonologie du français : normes, périphéries, modélisation, Paris, Presses universitaires de Paris Nanterre, p. 173-196

DIONNE, N.-E., 1912, Une dispute grammaticale en 1842 : le g.-v. Demers vs. le g.-v. Maguire : précédée de leur biographie, Québec, Laflamme \& Proulx.

DULONG, G., \& BERGERON, G., 1980, Le parler populaire du Québec et de ses régions voisines, Québec, Gouvernement du Québec, Ministère des communications/Office de la langue française.

DURAND, J., LAKS B., \& LYCHE, C., 2014, « French Phonology from a Corpus Perspective: The PFC Programme », dans J. Durand, U. Gut et G. Kristoffersen (éds.), The Oxford Handbook of Corpus Phonology, Oxford, Oxford University Press, p. 486-497.

DUMAIS, J., 1905, Parlons français. Petit traité de prononciation, [s.n.]

FRÉCHETTE, L., 1894, « À travers le dictionnaire et la grammaire. Corrigeons-nous ! », La Patrie, 26 mai 1894, Montréal.

FRENETTE, Y., 2008, « Immigration et francophonie canadienne au tournant du XXIe siècle », dans D. Louder \& E. Waddel (éds.), Franco-Amérique, Québec, Septentrion, p. 345-354.

FLYNN, N. E. J., \& FOULKES, P. 2011, « Comparing vowel formant normalization methods », dans W. S. Lee \& E. Zee (eds.), Proceedings of the 17th ICPhS, Hong Kong, The City University of Hong Kong, p. 683-686.

HUDON, T.S.J., 1931, Manuel de Prononciation française, Imprimerie du Messager.

KEMP, W. \& YAEGER-DROR, M., 1991, « Changing realizations of A in ation in relation to the front a back a opposition in Québec French », dans P. Eckert (ed.), New Ways of Analyzing Sound Change, San Diego, Academic Press, p, 127-184.

LAMONDE, Y., 2000, Histoire sociale des idées au Québec : 1760-1896, Montréal, Éditions Fides.

LANDREAU, G., 1931, Les Éléments de Phonétique Française, [s.n.]

MAGUIRE, T., 1841, Manuel des difficultés les plus communes de la langue française, adapté au jeune âge : et suivi d'un recueil de locutions vicieuses, Québec, Fréchette \& cie.

MANSEAU, J. A., 1881, Dictionnaire des locutions vicieuses du Canada, avec leur correction : suivi d'un dictionnaire canadien, Québec, J.A. Langlais.

MILNE, P., 2012, « The Effects of Syllable Position on Allophonic Variation in Quebec French /R/ ", University of Pennsylvania Working Papers in Linguistics, vol. 18, n² 2, p. 67-76. 
MORIN, Y.- C., 2002, « Les premiers immigrants et la prononciation du français au Québec », Revue québécoise de linguistique, vol. 31, $\mathrm{n}^{\circ}$ 1. p. 39-78.

MORIN, Y.- C., 2009, « À propos de la fermeture des voyelles moyennes devant [r] dans le français du Québec », Revue canadienne de linguistique, vol. 54, n 3, p. 461-510

PARIS, F., 1901, « Causerie philologique », La Défense, 21 mars 1901, Chicoutimi.

PASQUES, L., 1975, «L'ancienne diphtongue oi », Romania, n 381, p. 67-82.

RINFRET, R., 1896, Dictionnaire de nos fautes contre la langue française, Montréal, Cadieux \& Derome Éditeurs.

RIVARD, A., 1901, Manuel de la parole - Traité de prononciation, Québec, J.-P. Garneau LibraireÉditeur.

SAINT-AMANT LAMY, H., 2016, « Kilomètre zéro : /R/ et le statut épicentral de Montréal dans le domaine linguistique laurentien », Cahiers internationaux de sociolinguistique, vol. 10, p. 101-134.

SAINT-AMANT LAMY, H., 2020, « Un siècle à haute voix : aspects temporels et géographiques du changement de timbre des voyelles pré-rhotiques en français laurentien », dans D. Bigot, D. Liakin, R. Papen, A. Jebali et M. Tremblay (éds.), Les français d'ici en perspective, Québec, Presses de l'Université Laval, p. 51-82.

TARDIVEL, J.-P., 1901, La langue française au Canada, Montréal, La Revue canadienne.

THUROT, C., 1881, De la prononciation française depuis le commencement du XVIe siècle : d'après les témoignages des grammairiens (volume 1), Paris, Imprimerie nationale.

TRUDGILL, P., 1974, « Linguistic change and diffusion: Description and explanation in sociolinguistic dialect geography ", Language in society, vol. 3, n 2, p. 215-246.

\section{NOTES}

1. Je tiens à remercier Marie-Hélène Côté pour les discussions ayant guidé la mise en place et la réalisation du projet de recherche duquel est tirée la présente étude. Je remercie également les deux lecteurs anonymes qui ont généreusement offert leurs commentaires sur la première version de cet article. J'assume évidemment l'entière responsabilité pour toute erreur potentielle dans les données ou l'analyse.

2. En raison de son timbre distinct, le $/ \varepsilon /$ long du français québécois est noté par le symbole [3]. De son côté, la voyelle basse postérieure est considérée arrondie, d'où l'utilisation du symbole [D]. Voir Côté (2012) pour une explication de ces choix typographiques.

3. Palsgrave (153), Sylvius (1531), Meigret (1542), Péletier (1549), Périon (1555), Pillot (1559), Ramus (1562), Cauchie (1570), Baiff (1574), Saint-Liens (1580), Bèze (1584), Martin (1632), Oudin (1633), Fremont d'Ablancourt (1645), Ménage (1672), d'Aisy (1674), Hindret (1687), Buffier (1709), Dumas (1733), Antonini (1753), Mauvillon (1754) et Douchet (1762).

4. Lubin (1609), Van der Aa (1622), Raillet (1668), de Pratel (1689), Milleran (1692), Mauger (1697), Bouchot (1759), Féraud (1760), Syllabaire de Bouillon (1777), Pielat (1799) et Domergue (1805).

5. Il n'est pas clair ici si Durand réfère à une voyelle intermédiaire entre $[\varepsilon]$ et [a] (c.-à-d. [æ]), ou entre $[\varepsilon]$ et $[\alpha]$ (un timbre s'approchant d'un schwa ou de [e]).

6. Meigret (1542), Péletier (1549), Baiff (1574), Estienne (1582), Lanoue (1596), Du Val (1604), Corneille (1687), Anonyme (1689), De Soule (1698), Regnier (1705), Billecoq (1711), d'olivet (1736), Demandre (1769). 
7. Joubert (1579), Girard (1716), Chalons (1716), Voltaire (1739), Boindin (1753), Académie française (1762), Douchet (1762).

8. La valeur à $40 \%$ de la durée de la voyelle correspond grosso modo au noyau des variantes diphtonguées des voyelles pré-rhotiques. Le script développé par Mielke est disponible au https://phon.wordpress.ncsu.edu/lab-manual/scripts/script-repository/.

9. Selon l'étude comparative de Flynn et Foulkes (2011), la procédure de normalisation proposée par Bladon et al. (1984) réduit significativement, par rapport à l'échelle hertzienne, la variabilité induite par les différences physiologiques entre les locuteurs tout en préservant la variabilité d'origine dialectale ou sociale. Cette méthode dite "intrinsèque " (où chaque observation est considérée séparément) a l'avantage de pouvoir être employée en l'absence d'une description complète du système sonore de chaque témoin.

10. Les distances de Mahalanobis permettent d'évaluer les observations de façon holistique plutôt que par un traitement séparé pour chaque formant. Elles ont l'avantage, par rapport aux distances euclidiennes, de rendre compte de la variance observée dans chacune des dimensions considérées (ici, les trois premiers formants).

11. Les voyelles appartenant à la catégorie /e/ sont le reflet des anciens /e/ paroxytoniques du français. La catégorie comprend les mots ayant la graphie -(i)ère, ainsi que le verbe faire et ses dérivés (ex. défaire, affaire, etc.)

12. L'analyse acoustique de la diphtongue /wo/ s'est faite sur sa partie stable (noyau) seulement. La partie glissante de la diphtongue n'a pas été analysée.

13. La différence observée entre les milieux de basse et de moyenne densité avant 1900 n'est pas significative.

14. La cohorte 1872 - 1909 a dû être écartée de la régression puisque l'information sur le nombre d'années d'étude précis des témoins n'est pas connue.

15. Les niveaux d'instruction correspondent aux nombres d'années de scolarité suivants: primaire [ 6 ans ou moins], secondaire [7 à 11 ans], collégial [12 à 13 ans], universitaire [14 ans ou plus].

16. Les valeurs de F2 plus élevées chez les universitaires des villes de taille moyenne de la cohorte 1980 - 1997 sont difficiles à interpréter. Il pourrait s'agir d'un résultat aberrant associé au petit nombre de témoins pour cette catégorie $(\mathrm{N}=4)$ ou d'une tendance réelle mal comprise. Les limites de l'échantillon ne permettent malheureusement pas d'explorer la seconde hypothèse en profondeur.

17. À partir de 1841, l'évêque de Montréal, Mgr Ignace Bourget, fait venir sept communautés religieuses françaises dont les membres occuperont des postes clés dans les paroisses, missions et écoles du Québec (Frenette, 2008). Le rôle de ces nouveaux arrivants sur l'adoption des changements n'est toutefois pas sans équivoque. L'attachement du clergé aux valeurs et, vraisemblablement pour un certain nombre de ses membres, aux usages linguistiques, de l'Ancien Régime aurait également pu contribuer au maintien des formes traditionnelles.

\section{RÉSUMÉS}

Cet article documente l'abandon, au Québec, de deux traits conservateurs du système sonore du français: la variante antérieure de la diphtongue -oi (ex. poire [pwєb]) et le maintien d'un contraste phonémique entre /e/ et $/ \varepsilon / \operatorname{devant} / \mathrm{s} /$ (ex. père $[\mathrm{pe}]$, paire $[\mathrm{p \varepsilon} \mathrm{s}]$ ). Ces deux traits, 
critiqués dans le discours normatif français dès les $17^{\mathrm{e}}$ et $18^{\mathrm{e}}$ siècles, auront persisté au Québec jusqu'au tournant du $21^{\mathrm{e}}$ siècle, rendant possible une analyse acoustique de leur déclin. À partir de données orales recueillies auprès de 152 témoins nés entre 1872 et 1997, une chronologie des processus est établie et les facteurs les conditionnant sont identifiés. Les résultats obtenus suggèrent que l'abandon des deux traits de prononciation commence vers le milieu du $19^{\mathrm{e}}$ siècle, au moment où l'intelligentsia canadienne-française, après sept décennies d'isolement, reprend contact avec les normes linguistiques hexagonales. Les schémas géographiques qui émergent des données mettent en lumière le rôle non-négligeable qu'un discours normatif explicite peut avoir sur le rythme de diffusion de nouvelles formes de prestige et l'abandon de formes anciennes tombées en défaveur.

This paper provides an account of the downfall, in Quebec, of two conservative aspects of the sound system of French: the anterior variant of the diphthong -oi (e.g., poire [рwєr]) and the retention of a phonemic contrast between /e/ and / $\varepsilon /$ before /ь/ (е.g., père [рев], paire [рєь]). These two features, criticized in the French normative discourse as early as the 17th and 18th centuries, have persisted in Quebec up to the turn of the 21st century, making an acoustic analysis of their decline possible. On the basis of oral data collected from 152 speakers born between 1872 and 1997, a chronology of the processes is established and the factors underlying them are identified. The results suggest that the decline of the two features begins around the middle of the 19th century, when the French-Canadian intelligentsia, after seven decades of isolation, reconnects with French linguistic norms. The geographic patterns emerging from the data highlight the non-negligible role that explicit normative discourse can have on the rate of diffusion of new prestige variants and the demise of those that have fallen into disfavour.

\section{INDEX}

Mots-clés : français québécois, diffusion des normes de prononciation, diphtongue -oi, contraste [e- $\varepsilon$ ], géophonologie, étude longitudinale

Keywords : Quebec French, diffusion of pronunciation standards, diphthong -oi, [e- $\varepsilon$ ] contrast, geophonology, longitudinal study

\section{AUTEUR}

\section{HUGO SAINT-AMANT LAMY}

Université de Lausanne 